\title{
Galaxy properties in clusters: dependence on the environment and the cluster identification techniques ${ }^{\star}$
}

\author{
V. Coenda ${ }^{1,2}$ and H. Muriel ${ }^{1,2}$ \\ 1 Instituto de Astronomía Teórica y Experimental IATE, CONICET, Laprida 922, X5000BGR, Córdoba, Argentina \\ e-mail: [vcoenda; hernan]@.mail.oac.uncor.edu \\ 2 Observatorio Astronómico, Universidad Nacional de Córdoba, Laprida 854, X5000BGR, Córdoba, Argentina
}

Received 11 December 2008 / Accepted 10 June 2009

\section{ABSTRACT}

\begin{abstract}
Aims. We investigate the dependence of several galaxy properties on the environment and cluster identification techniques. Methods. We select clusters of galaxies from two catalogues based on the SDSS: the ROSAT-SDSS galaxy cluster survey, which is an X-ray selected cluster sample and the MaxBCG catalogue, in which clusters are optically selected. Based on a volume limited sample of galaxies drawn from the spectroscopic DR5 SDSS, we constructed sub-samples of clusters of galaxies with more than ten members. Scaling relations as well as segregation of galaxy properties as a function of the normalized clustocentric radii are analyzed. The properties of galaxies in clusters are compared with those of field galaxies.

Results. Galaxies in X-ray and MaxBCG selected clusters show similar size-luminosity relations. At equal luminosity, late type galaxies in the field have sizes smaller than cluster galaxies of the same morphological type.

The Faber-Jackson relation for early-type galaxies in clusters is also the same for X-ray selected and MaxBCG clusters. We found clear differences between the dynamical properties of galaxies in clusters, the brightest cluster galaxies $\left(B C G_{\mathrm{s}}\right)$ and field galaxies. Using several criteria to classify galaxies into morphological types, we reproduce the well know morphological segregation. The correlation is up to $r / r_{200} \sim 1$. For the whole range of clustocentric distances, X-ray selected clusters present a higher fraction of early type galaxies than MaxBCG clusters. We also found that bright galaxies preferentially inhabit the cluster centers. Median sizes of galaxies, such as the radius that encloses $50 \%$ of Petrosian flux $r_{50}$, present a behaviour that also depends on the cluster selection criteria. For galaxies in X-ray selected clusters, median values of $r_{50}$ decrease as $r / r_{200}$ goes to zero, whereas the opposite is observed for galaxies in the MaxBCG clusters. These different behaviours are mainly due to early type galaxies.

The results are discussed in terms of the different processes that affect the evolution of galaxies in different environments.
\end{abstract}

Key words. galaxies: clusters - catalogs - general - galaxies: fundamental parameters - X-rays: galaxies: clusters

\section{Introduction}

It is well known that galaxies show a wide range of morphologies, which can be appreciated in their properties such as colour, luminosity, size, star formation histories, etc., which imply that galaxies form and evolve through different mechanisms. There have been many studies about the distribution of galaxies with respect to their properties. For example, the luminosity function has been measured from various surveys of galaxies and clusters of galaxies and it is found to be well described by the Schechter function (Schechter 1976; Loveday et al. 1992; Folkes et al. 1999; Madgwick et al. 2002; Cross et al. 2004); the morphological types of galaxies are found to be correlated with the environment, clusters of galaxies being the best example (Dressler 1980; Dressler et al. 1997; Domínguez et al. 2001; Coenda et al. 2006). It is also well known that different galaxy properties are correlated to each other. Galaxy sizes are correlated with the luminosity and morphological type (Andredakis et al. 1995; Möllenhoff \& Heidt 2001; Coenda et al. 2005; McIntosh et al. 2005; Trujillo et al. 2006), and have a distribution that may be described by a log-normal function (Syer et al. 1999; de Jong \& Lacey 2000; Shen et al. 2003; Ferguson et al. 2004). In order to constrain the galaxy formation models and the study of galaxy properties and

\footnotetext{
* Appendix A is only available in electronic form at http://www . aanda. org
}

their dependence on environment, we concentrate our studies on several scaling relations and galaxy segregation.

There are different scaling relations between photometric and structural parameters of galaxies, resulting in well known relations. One of the most established empirical scaling relations of disk galaxies is the Tully-Fisher relation (Tully \& Fisher 1977), which consist of a correlation between luminosity and rotational velocity. The analogous relation for spheroidal galaxies is the correlation between the velocity dispersion of bulges and luminosity, known as the Faber-Jackson relation: $L \propto \sigma^{\beta}$ (Faber \& Jackson 1976). Other scaling relations for spheroidal galaxies are: color-magnitude (Sandage \& Visvanathan 1978a,b), colorvelocity dispersion (Bernardi et al. 2005), radius-luminosity (Sandage \& Perelmuter 1990) and the Kormendy relation, which is a correlation between radius and surface brightness (Kormendy 1977). Some of these properties have been combined to define the so-called fundamental plane that relates the velocity dispersion, the effective radius and the luminosity (Djorgovski \& Davis 1987; Dressler et al. 1987; Bernardi et al. 2003). These empirical relations are closely related to the physical processes involved in the galaxy formation scenario and, therefore, are a fundamental tool to understand the formation and evolution of galaxies. These relations could depend on the environment where galaxies form and evolve, introducing departures from scatter in the different scaling relations. Several authors have 
investigated the scaling relations of galaxies in clusters. Ziegler et al. (1999), La Barbera et al. (2004), Bernardi et al. (2007), von der Linden et al. (2007), Liu et al. (2007) and Bildfell et al. (2008). von der Linden et al. (2007) found that brightest cluster galaxies (BCG) have a higher fraction of dark matter and consequently larger radii and higher velocity dispersions that nonBCG galaxies. Malumuth \& Kirshner (1981, 1985), Oegerle \& Hoessel (1991) and more recently Bernardi et al. (2007) found that BCG have smaller $\beta$ than the rest of the cluster members. Bernardi et al. (2007) also analyze the dynamical mass $\left(M_{\text {dyn }} \propto r_{50} \sigma^{2}\right)$ and found that BCGs show a steeper relation than non-BCG galaxies. The majority of the works mentioned above do not make a suitable comparison with field galaxies, making it very difficult to perform a complete comparison of the scaling relations of galaxies as a function of the environment. More recently, Weinmann et al. (2009) and Guo et al. (2009) analyzed samples of galaxies in groups and found that, at fixed stellar mass, the sizes of central and satellite early type galaxies are similar, while Bernardi (2009), analyzing galaxies in clusters, reported a difference at high luminosities/stellar masses between central and satellite galaxies.

It is well known that different types of galaxies show different spatial distributions (Oemler 1974; Melnick \& Sargent 1977; Dressler 1980). These differences can be estimated measuring the radial dependence of the galaxy properties as a function of the clutocentric distance. The segregation of galaxies in clusters has been quantified splitting galaxies according to different properties, with the morphology the property that has been most extensively studied, see for instance Dressler (1980), Whitmore et al. (1993), Domínguez et al. (2001), Biviano et al. (2002), Driver et al. (2003), Coenda et al. (2006). Goto et al. (2002) found that the morphological segregation tends to disappear for clustocentric distances larger than the virial radius. Recently, Martínez et al. (2008) found that $g-r$ color is the property of galaxies that best predict the normalized clutocentric distance of galaxies among a set of galaxy properties. Similarly, Skibba et al. (2008) used the galaxy sample of visual classified morphologies and found that much of the morphology-density relation is due to the relation between colour and density. The works that have studied the segregation in luminosity present some contradictory results. Rood \& Turnrose (1968), Yepes et al. (1991), Lobo et al. (1997), Kashikawa et al. (1998), Coenda et al. (2006) found results consistent with a segregation in luminosity. Nevertheless, Noonan (1961), Bahcall (1973) and more recently Pracy et al. (2005) found results consistent with a lack of segregation in luminosity. The segregation of other properties like color, star formation rate, gas content or structural parameters have been much less studied.

The identification of clusters of galaxies is a complex process whose outcome depends on the selection criteria. Popesso et al. (2004) compiled X-ray clusters in the Sloan Digital Sky Survey (SDSS). Using the same survey, Koester et al. (2007b) applied a maxBCG algorithm to identified clusters. This technique is based on the color-magnitude relation of the early type galaxies and the properties of the BCGs. Because we are interested in comparing the galaxy properties of clusters selected with different criteria, the same analysis will be performed on sub-samples of the catalogues compiled by Popesso et al. (2004) and Koester et al. (2007b).

This paper is organised as follows: in Sect. 2 we describe the cluster and galaxy samples; in Sect. 3 we investigate several galaxy scaling relations; in Sect. 4 we analyze the galaxy segregation. We summarise and discuss our results in Sect. 5 .

\section{The sample}

\subsection{The cluster sample}

We use two catalogues of clusters of galaxies based on the Sloan Digital Sky Survey (York et al. 2000): the ROSAT-SDSS galaxy cluster survey of Popesso et al. (2004, hereafter P04), which is a X-ray selected cluster sample and the MaxBGC Catalogue of Koester et al. (2007b, hereafter K07), which is an optically selected cluster sample.

The ROSAT-SDSS catalogue of Popesso et al. (2004) comprises 114 galaxy clusters detected in the ROSAT All Sky Survey (RASS) lying in the area surveyed by the SDSS by February 2003. This X-ray-selected catalogue includes clusters with masses from $10^{12.5} M_{\odot}$ to $10^{15} M_{\odot}$ in the redshift range $0.002 \leq z \leq 0.45$. This catalogue provides X-ray properties of the clusters derived from the ROSAT data, parameters of the galaxy luminosity function and the luminosity of each cluster computed from SDSS data, and the radial distribution of the projected galaxy density around clusters. Since the X-ray observations provide a robust method for identifying clusters, these clusters constitute a reliable source of information to study galaxy properties and their correlation with the environment.

The optical MaxBGC catalogue provides sky locations, photometric redshift estimates and richness for 13823 clusters. Details of the selection algorithm and catalogue properties are published in Koester et al. (2007a,b) The MaxBGC selection relies on the observation that the galaxy population of rich clusters is dominated by bright red galaxies tightly clustered in colour (the E/S0 ridgeline). Since these galaxies are old, passively evolving stellar populations, their $g-r$ colours closely reflect their redshifts. The brightest red galaxy, typically located at the peak of the galaxy density, defines the cluster centre. The K07 catalogue comprises galaxy clusters with velocity dispersions $\sigma \geq 400 \mathrm{~km} \mathrm{~s}^{-1}$ and redshifts $0.1 \leq z \leq 0.3$. Each of these clusters contains between 10 and $190 \mathrm{E} / \mathrm{S} 0$ ridgeline galaxies brighter than $0.4 L_{*}$ ( $i$-band), within a scaled radius $r_{200}$ defined as the mean density of 200 times the mean density of the universe. SDSS spectroscopic redshifts are available for at least the brightest galaxy in $39 \%$ of the clusters. The sample is up to $85 \%$ complete for clusters with masses $\geq 10^{14} M_{\odot}$.

The sub-samples from P04 and K07 considered in this paper comprise galaxy clusters in the redshift range $0.05<z<0.14$. The upper limit was taken in order to have a volume limited sample of galaxies with an adequate balance between the number of clusters, the range in absolute magnitude and the number of cluster members. For K07 clusters, we applied a restriction in the richness, selecting clusters with $N_{\text {gal }} \geq 20$ in order to have cluster masses comparable to those in the P04 sample. The subsamples are labeled as C-P04 and C-K07 respectively. To select cluster members and estimate the physical properties of clusters, we use the main galaxy sample (MGS, Strauss et al. 2002) of the fifth data release (DR5) of SDSS (Adelman-McCarthy et al. 2007) that is complete down to a Petrosian (1976) magnitude $r=17.77$. At this point, our sub-samples comprise 54 X-ray galaxy clusters from the P04 sample and 612 from the K07 sample. We show in panel (a) of Fig. 1 the spectroscopic redshift distribution of 114 galaxy clusters of the P04 sample (grey solid line), the photometric redshift distribution of 13823 galaxy clusters of the K07 sample (black solid line), the distributions of those K07 clusters with $N_{\text {gal }} \geq 20$ (dotted line) and the distribution of those K07 clusters with spectroscopic redshifts (dashed line). Panel (b) shows the redshift distributions of the selected clusters C-P04 (grey line) and C-K07 (black line). We cross-correlated the catalogues to pick clusters that are included 


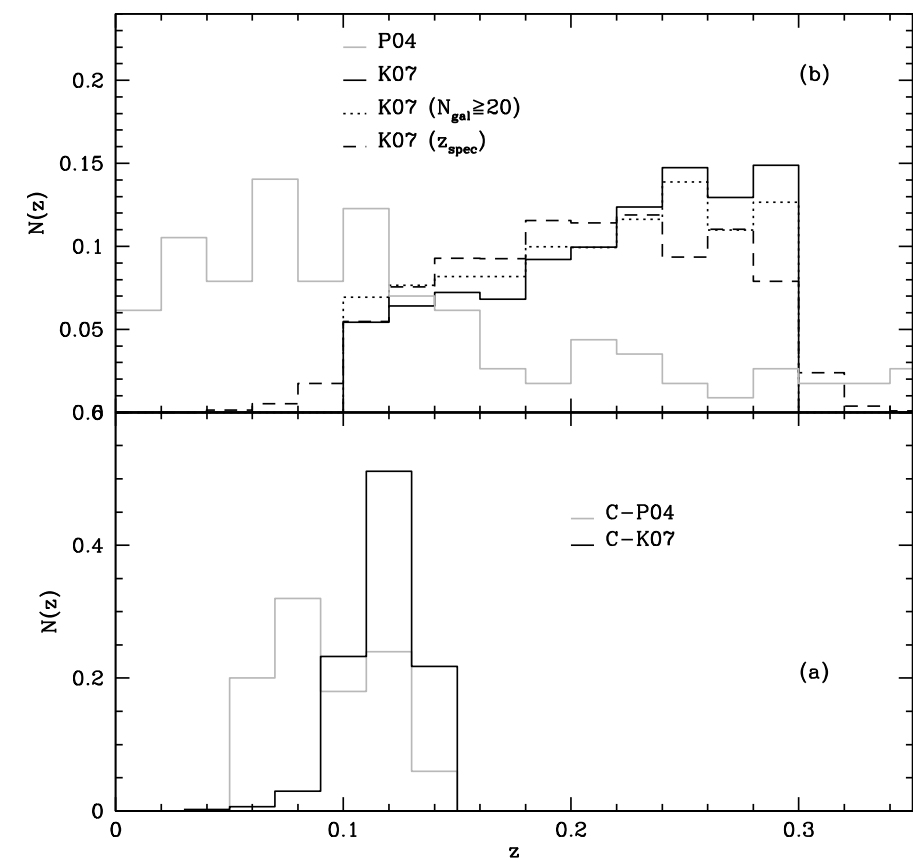

Fig. 1. Panal a) shows the spectroscopic redshiht distribution of the P04 galaxy clusters (grey solid line), the photometric redshift distribution of the K07 galaxy clusters (black solid line), the distributions of those K07 clusters with $N_{\text {gal }} \geq 20$ (dotted line) and the distribution of those K07 clusters with spectroscopic redshifts (dashed line). Panel b) shows the redshift distributions of the selected clusters C-P04 (grey line) and C-K07 (black line).

in both of them. If the projected distance between 2 clusters each belonging to a different catalogue is $<1 \mathrm{Mpc}$, we consider that they are the same cluster. We found 20 coincidences; in these cases the clusters are only included in the X-ray sub-sample (C-P04).

In order to compute cluster properties such as velocity dispersion, we first identified the cluster members. We use SDSS galaxies from the MGS within a projected radius of $3 \mathrm{Mpc}$ centred on the quoted cluster coordinates. From these galaxies we identify cluster members in two steps. First, we use the friendsof-friends (fof) algorithm developed by Huchra \& Geller (1982) with the percolation linking length values according to Díaz et al. (2005). As a result, we get for each field a list of substructures with at least 10 members identified by fof. The second step consists of eyeball examination of the structures detected by fof, a comparison between them and the listed cluster position and redshift to determine which coordinates and redshift fit better the observed galaxy over-density. From the redshift distribution of galaxies within $\left|c z-c z_{\text {cluster }}\right| \leq 3000 \mathrm{~km} \mathrm{~s}^{-1}$ we determine the line-of-sight extension of each cluster, i.e., a maximum and a minimum redshift for the cluster. We then consider as cluster members all galaxies in the field that are within that redshift range. The Díaz et al. (2005) technique includes an iterative method that provides precise dynamical centres of the clusters. When these centres differ from those computed by Koester et al. (2007a), we adopted the fof values. We found that for $\sim 40 \%$ for the clusters, the angular position given by the fof is different from the original value, whereas for $\sim 17 \%$ of the clusters the redshift derived from the fof algorithm is a better match to the observed galaxy redshift distribution than those quoted in the original catalogues. Finally, we excluded from our sample those clusters for which the fof redshift value lay outside the redshift range we consider in this paper.
Table 1. Mean values of the cluster physical properties of our cluster samples.

\begin{tabular}{ccccc}
\hline \hline Sample & $\begin{array}{c}\sigma \\
{\left[\mathrm{km} \mathrm{s}^{-1}\right]}\end{array}$ & $\begin{array}{c}R_{200} \\
{\left[h^{-1} \mathrm{Mpc}\right]}\end{array}$ & $\begin{array}{c}M_{\text {vir }} \\
{\left[h^{-1} M_{\odot}\right]}\end{array}$ & $\begin{array}{c}R_{\text {vir }} \\
{\left[h^{-1} \mathrm{Mpc}\right]}\end{array}$ \\
\hline C-P04-I & 715 & 1.77 & $7 \times 10^{14}$ & 1.75 \\
C-K07-I & 675 & 1.67 & $6 \times 10^{14}$ & 1.59 \\
\hline
\end{tabular}

Once the members of each cluster are selected, we compute cluster physical properties we are interested in. We compute the line-of-sight velocity dispersion $\sigma$, the virial radius and mass and the $r_{200}$. The line-of-sight velocity dispersion $\sigma$ is estimated using methods described by Beers et al. (1990). The bi-weight estimator was applied to clusters with $\geq 15$ members, whereas the gapper estimator was applied to poorer clusters. The radius $r_{200}$ was computed using the approximation provided by Carlberg et al. (1997). In a first step, we determined these parameters using all the cluster members within a $3 \mathrm{Mpc}$ projected distance. After this step, we recalculated $\sigma$ and $r_{200}$ using only galaxies located inside $r_{200}$. Virial radis and masses were computed following Merchán \& Zandivarez (2005). The mean values of these parameters are shown in Table 1, where it can be seen that the sample C-P04 includes on average clusters slightly more massive and larger than the sample C-K07. Figure 2 shows the distributions of cluster physical properties of our samples. The distributions of the main properties of the 20 coincident clusters are similar to those of the parent samples.

Through visual inspection we classified clusters based on their substructure. We only consider in our analysis sub-samples of regular clusters, that we label C-P04-I and C-K07-I, and we exclude systems that have two or more close substructures of similar size in the plane of the sky and/or in the redshift distribution. In order to compute the physical properties in a reasonable way, clusters with fewer than 10 galaxy members within $r_{200}$ are excluded (Girardi et al. 1993). Our final galaxy cluster sample comprises 49 clusters from C-P04-I and 209 from C-K07-I. Our sample of MaxBCG clusters is an order of magnitude smaller than the sample considered by Bernardi (2009), also based on the Koester et al. (2007a) catalogue. This difference is mainly due to our restriction to select clusters with at least ten spectroscopic members in the redshift range $0.05<z<0.14$. The sample of Bernardi (2009) includes clusters with redshift up to 0.3 and has, on average, fewer than one spectroscopic early-type satellite galaxy per cluster.

\subsection{The galaxy sample}

In order to avoid the use of weights and since we are interested in bright galaxies, we have constructed a volume limited samples instead of a flux limited sample. Taking into account the depth of our sample $(z<0.14)$ and the apparent magnitude limit of the Main Galaxy Sample of the SDSS, our sample of galaxies only includes objects with $M_{0.1}<-21.3$. The C-P04-I sample comprises 786 galaxies, while the C-K07I sample has 3041 galaxies. Figure 3 shows the redshift distributions of galaxies of our samples, C-P04-I clusters with the grey line and C-K07-I sample with the black line. Galaxy magnitudes used throughout this paper have been corrected for galactic extinction following Schlegel et al. (1998), absolute magnitudes have been computed assuming $\Omega_{0}=0.3, \Omega_{\Lambda}=0.7$ and $H_{0}=70 h \mathrm{~km} \mathrm{~s}^{-1} \mathrm{Mpc}^{-1}$ and $K$-corrected using the method of Blanton et al. (2003) (KCORRECT version 4.1). All magnitudes are in the $\mathrm{AB}$ system. 

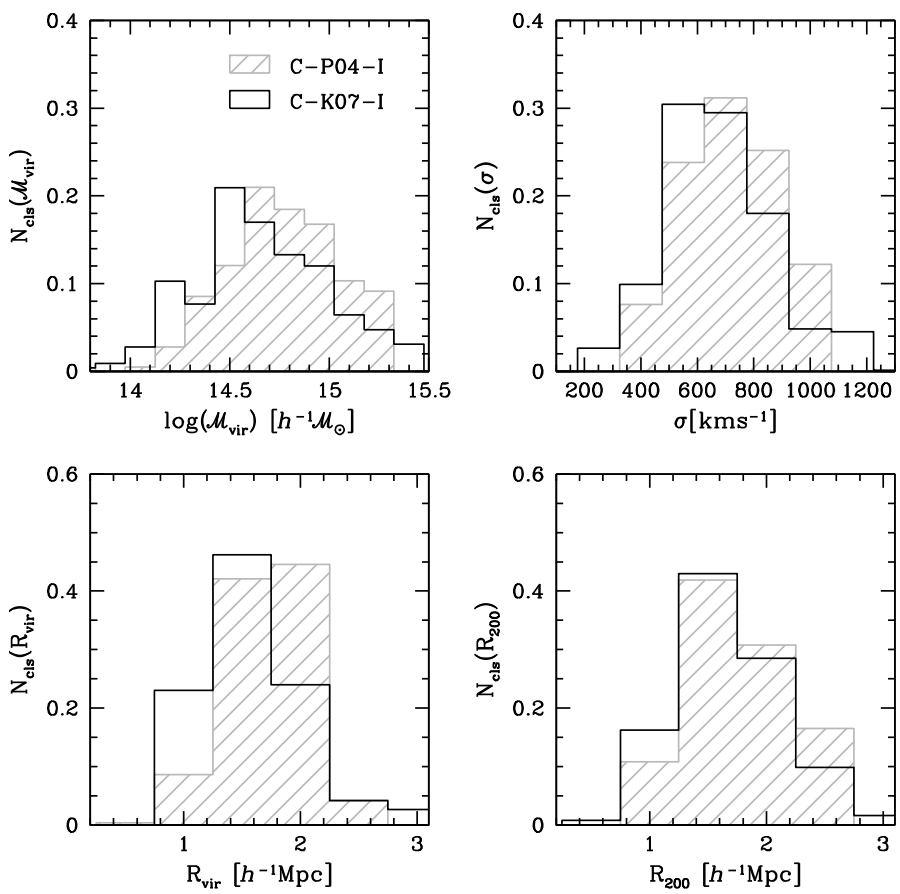

Fig. 2. Distributions of cluster physical properties. The solid grey lines show the C-P04-I sample while the dashed histograms correspond to the C-K07-I sample.

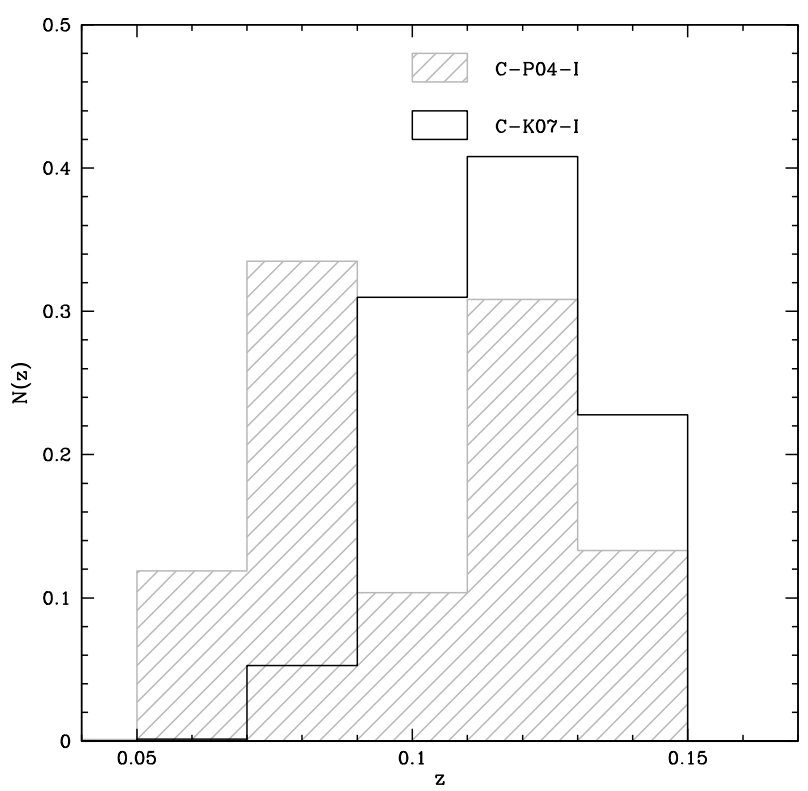

Fig. 3. Redshift distributions of galaxies in our samples. Grey line: C-P04-I sample, black line: C-K07-I sample.

Among the available data for each object in the MGS, we have selected for our analyses parameters that are related to different physical properties of galaxies: luminosity, star formation rate, light distribution inside the galaxies and the dominant stellar populations. In particular, the galaxy parameters we have focused our study on are the Petrosian $r$-band absolute magnitude $M_{0.1} r$ and the radius that encloses $50 \%$ of the Petrosian flux $r_{50}$. We have chosen Petrosian quantities that are not corrected for the effects of seeing. In order to reduce the effect of the PSF on galaxy sizes, we excluded galaxies with $r_{50}<2^{\prime \prime}$, i.e. $0.5^{\prime \prime}$ greater than the average seeing in SDSS. This is a conservative threshold, since the PSF in SDSS is known quite accurately
(Shen et al. 2003). Hyde \& Bernardi (2009) analysed residuals from the size-luminosity relation in the SDSS Petrosian $r$-band in different redshift ranges. As expected, they found that the effect of seeing increases as a function of redshift. However, in our redshift range $(0.05<z<0.14)$, these residuals are close to 0 .

Our sample consists of bright galaxies in crowded fields where SDSS reductions tend to overestimate the sky level. This results in underestimates of magnitudes and half-light radius for large half-light radii galaxies (Adelman-McCarthy et al. 2008; Bernardi et al. 2007; Hyde \& Bernardi 2009). In order to correct the magnitudes and sizes by sky level, we have fitted curves to SDSS simulations (DR6 documentation) and we used these fits to correct the SDSS reductions. Briefly, SDSS quantified the sky effect by adding simulated galaxies (with exponential or de Vaucouleurs) profiles to SDSS images. The simulated galaxies ranged from apparent magnitude $r=12$ to $r=19$ in halfmagnitude steps, with a one-to-one mapping from $r$ to Sérsic half-light radius (Sérsic 1963, 1968) determined using the mean observed relation between these quantities for MGS with exponential and de Vaucouleurs profiles. Axis ratios of 0.5 and 1 were used, with random position angle for the non-circular simulated galaxies. They found a difference between the input magnitude and the model magnitude returned by the SDSS photometric pipeline, as a function of magnitude. Their results are consistent with a separate analysis performed by Hyde \& Bernardi (2009). Finally, we have estimated the rest-frame radius $r_{50}$ in $r$-band interpolating the observed radii in the adjacent $i$-band as in Hyde \& Bernardi (2009).

Since the aim of this work is to analyze scaling relations and segregation of different types of galaxies in clusters and its comparison with field galaxies, we adopt several criteria to classify galaxies. Shimasaku et al. (2001) found that the concentration index shows a strong correlation with morphological type. These authors conclude that $C$ is perhaps the best parameter to classify the morphology of galaxies. They also found that galaxies with $R_{50}<2^{\prime \prime}$ show a weaker correlation between $C$ and the morphological type. To separate galaxies into early and late types, we use the $r$-band concentration index defined as the ratio between the radii that enclose $90 \%$ and $50 \%$ of the Petrosian flux, $C=r_{90} / r_{50}$. Typically, early-type galaxies have $C>2.5$, while for late-types $C<2.5$ (Strateva et al. 2001). We also use the color to discriminate between early and late type galaxies. The corresponding threshold is ${ }^{0.1}(g-r)=0.8-0.03\left(M_{r}+20\right)$ (see Blanton \& Berlind 2007). Other indicator of galaxy type is the mono-parametric spectral classification based on the eigentemplate expansion of galaxy's spectrum eclass. This parameter ranges from about -0.35 for early-type galaxies to 0.5 for late-type galaxies (Yip et al. 2004); the separation is set at eclass $=-0.1$. Finally, we use the Sérsic index $n$, taken from Blanton et al. (2005a). The distribution of $n$ shows a bimodal distribution (see for instance Ball et al. 2008; and Driver et al. 2006). The $n$ separation is set at 2.5 , the value that divides in equal parts the two distributions and corresponds to objects with approximately equal bulge and disk components. In Fig. 4 we show the distributions of galaxy parameters. We can see that clusters of the C-P04-I sample (on average, slightly more massive than C-K07-I) have a higher fraction of red galaxies than those taken from the C-K07-I sample (89\% and $85 \%$ respectively).

We are also interested in the study of the brightest cluster galaxies and the comparison with early-type galaxies in both clusters and in the field. We identify the spectroscopic brightest cluster galaxy $\left(B C G_{\mathrm{s}}\right)$ in our sample. The $B C G_{\mathrm{s}}$ in not necessarily the true BCG due to the following reasons: i) as a 

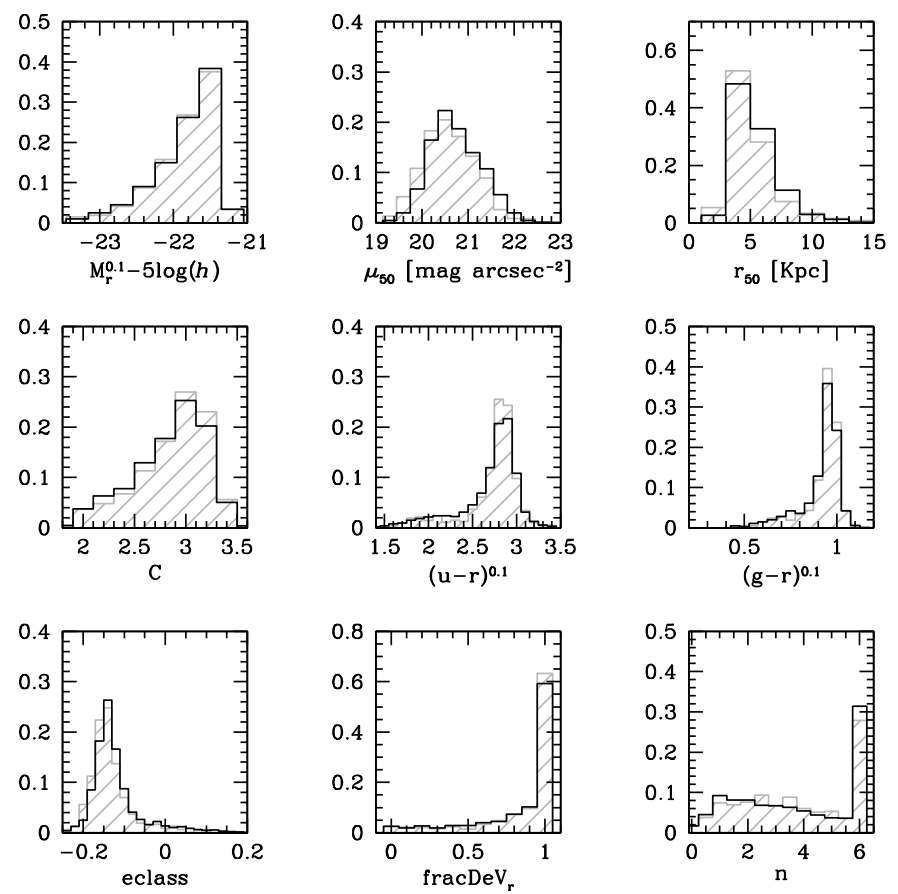

Fig. 4. Distributions of galaxy properties of our samples. Grey line: C-P04-I sample, black line: C-K07-I sample.

consequence of the finite fiber size of the SDSS spectrograph, any two fibers on the same plate need to be spaced at least $55^{\prime \prime}$ apart. In the case of a fiber collision, objects are selected at random; ii) the MSG is limited at the bright end by the fiber magnitude limits $(r=14.5)$, to avoid saturation and excessive crosstalk in the spectrograph. These two effects together cause the spectroscopic sample to become incomplete.

In Fig. 5 we show the distributions of $B C G_{\mathrm{s}}$ parameters. We find that $B C G_{\mathrm{s}}$ are larger and have lower surface brightness that non- $B C G_{\mathrm{s}}$, in agreement with von der Linden et al. (2007). In addition, $B C G_{\mathrm{s}}$ have higher velocity dispersion than non- $B C G_{\mathrm{s}}$. In general, we find that $B C G_{\mathrm{s}}$ have colors, concentration and eclass parameters typical of normal early-type galaxies. Despite that $B C G_{\mathrm{s}}$ are usually in the centre of the cluster potential well, we find that the $25 \%$ have $r / r_{200}>0.5$. Nevertheless, it is probable that a fraction of these $B C G_{\mathrm{s}}$ with large $r / r_{200}$ are not the actual BCG. It is also possible that some of the cluster centres are wrong. Finally, we do not find differences between the mean properties of $B C G_{\mathrm{s}}$ in the C-P04-I and C-K07-I samples.

To perform a suitable comparisson between cluster and field, we construct a set of comparison samples. These were drawn from MGS and consist of galaxies selected at random having the same redshift distribution as C-P04-I and C-K07-I. This procedure excludes the cluster members. For each cluster catalogue we have two types of samples: i) F-P04-T (52560 galaxies) and F-K07-T (51706 galaxies) that reproduce the corresponding total redshift distribution of galaxies in clusters and ii) F-P04-E, F-P04-L, F-K07-E and F-K07-L that reproduce the redshift distributions and concentration index distributions of the early and late type galaxy population of C-P04-I and C-K07-I, respectively.

\section{Scaling relations}

\subsection{Luminosity-size relation}

In this section we use the $r$-band concentration index $C$ to split galaxies into early and late types, as explained in Sect. 2.2.
In Fig. 6 we show the correlation between luminosity and the Petrosian half-light radius $\left(r_{50} \propto L^{\alpha}\right)$. We plot the median values of $\log \left(r_{50}\right)$ versus $M_{r}^{0.1}$ for the C-P04-I sample (top panels) and the C-K07-I sample (bottom panels). Error bars were obtained by the bootstrap re-sampling technique. Slopes and zero points in this work correspond to a least-square linear regression. The dotted lines show the $95 \%$ confidence bands of the linear regressions. Galaxies in X-ray and MaxBCG selected clusters show similar size-luminosity relations. Early and late type galaxies (red and blue lines respectively) show clear differences, late type galaxies being larger than early types for fixed luminosities. If clusters and field galaxies are compared, we can see in Fig. 6 and Table 2 that late type galaxies in the field (cyan lines) are smaller than galaxies in clusters for a fix luminosity.

For early type galaxies (magenta lines), the differences between field and cluster galaxies are also present, although, they are smaller. The effect is stronger for MaxBCG clusters. Finally, in the common range of luminosity, $B C G_{\mathrm{s}}$ (black lines) tend to be larger and show a steeper $\log \left(r_{50}\right)-M_{r}^{0.1}$ relation than non$\mathrm{BCG}$ early type galaxies in clusters. Except for $B C G_{\mathrm{s}}$, early type galaxies in clusters and in the field show the previously reported curvature in the size-luminosity relation (Bernardi et al. 2007). For these samples of galaxies, linear fits exclude the brightest luminosity bin.

We compare our results of the $r_{50}-M_{r}^{0.1}$ relation of BCGs with other studies. von der Linden et al. (2007) performed isophotal photometry from the $\mathrm{C} 4$ cluster catalogue (Miller et al. 2005) and they found a slope $\alpha=0.65 \pm 0.02$ for BCGs and $\alpha=0.63 \pm 0.02$ for a control sample. Liu et al. (2007) derived the luminosity-size relation for 85 BCGs from the same catalogue performing isophotal photometry down to four isophotal limits $\left(22,23,24\right.$ and 25 mag $\left.\operatorname{arcsec}^{-2}\right)$; they found that the luminosity-size relation become steeper as the isophotal limit becomes fainter for both BCGs and control sample galaxies. The slope varies between $0.63 \pm 0.04$ and $0.88 \pm 0.04$ for BCGs and between $0.58 \pm 0.04$ and $0.74 \pm 0.04$ for the control sample. Although these authors used a similar photometry to von der Linden et al. (2007), the difference in the slopes could be related to the differences in the brightness of the samples. Bernardi et al. (2007) fitted a de Vaucouleurs model to SDSS images from the $\mathrm{C} 4$ catalogue and used the half-light radius of their bestfitting models to study the luminosity-size relation. These authors found a slope $\alpha=0.89$. Lauer et al. (2007) used surface photometry in HST images for 219 early type galaxies and also fitted a de Vaucouleurs model; they found $\alpha=1.18 \pm 0.06$. Once again, the photometry used in the last two works is similar but the results are very different. Again, a suitable explanation is the difference in the construction of the samples. On the other hands, Guo et al. (2009) used 911 central galaxies from SDSS DR4 group catalogue (Yang et al. 2007). This catalogue is constructed using the New York University value-addes galaxy cata$\log$ (Blanton et al. 2005b). Guo et al. (2009) fitted a Sérsic luminosity profile to each galaxy using GALFIT (Peng et al. 2002), and found a slope $\alpha=1.02 \pm 0.03$ over the luminosity range -19 to -24 . For galaxies brighter than -22 , these authors found $\alpha=0.82 \pm 0.06$. Shen et al. (2003), using spectroscopic SDSS galaxies and also Sérsic luminosity profiles, found $\alpha=0.65$. Recently, Hyde \& Bernardi (2009) used early-type BCGs identified in the SDSS MaxBCGS and C4 catalogs and found $\alpha=0.6$ for early-type galaxies, while BCGs follow a steeper relation. It should be noted that the slopes resulting from our work are typically shallower than the slopes found in the different works mentioned above. Partially, these differences in slopes could be the result of the different methods used to perform the photometry. 

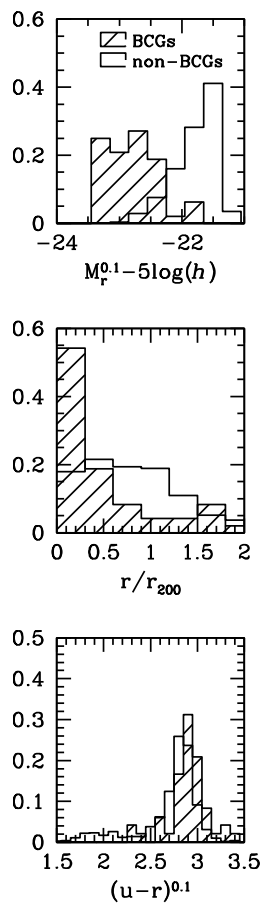
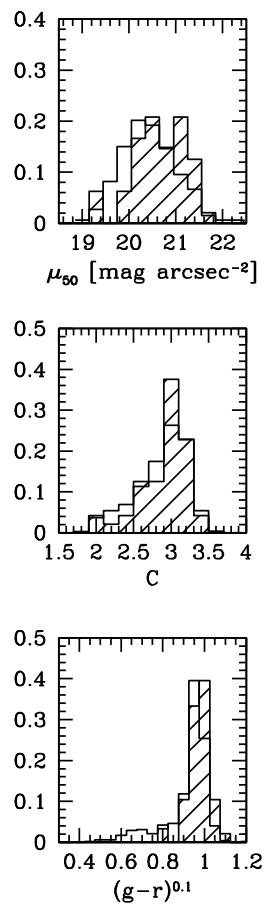
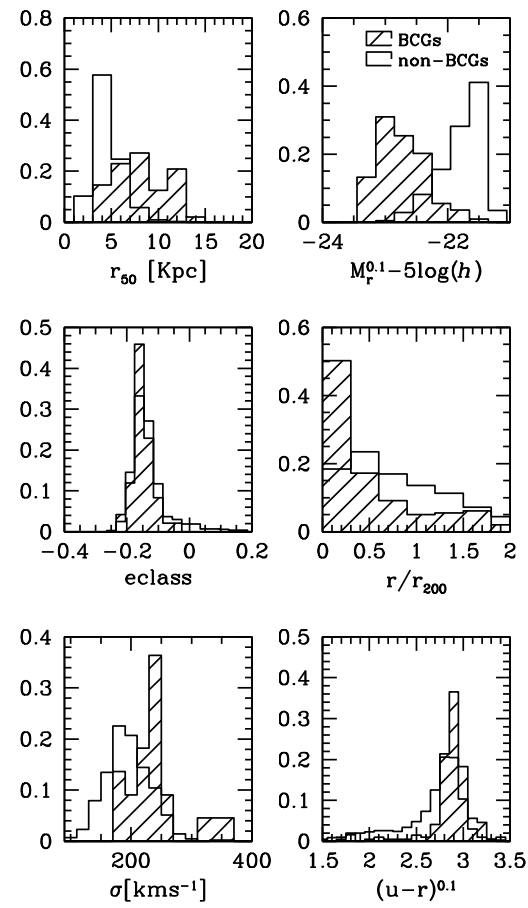
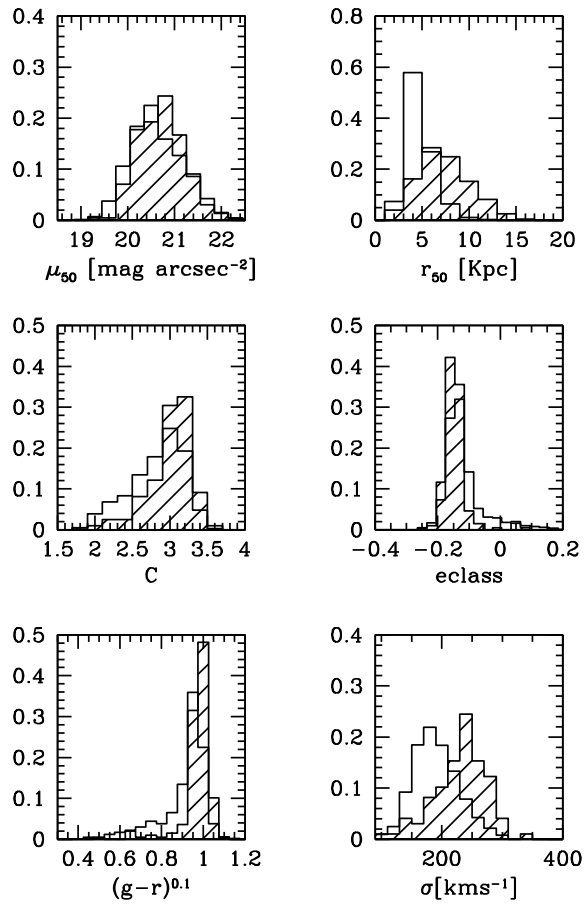

Fig. 5. Distributions of $B C G_{\mathrm{s}}$ (dashed histograms) and non - BCG galaxy properties of the samples C-P04-I (left panels) and C-K07-I (right panels).

Table 2. Parameter of the fits $\log \left(r_{50}\right)=-0.4 \alpha M_{r}+b$, see Fig. 6 .

\begin{tabular}{llll}
\hline \hline & C-P04-I & & \\
\hline & early non-BCGs & $B C G s$ & early field \\
\hline$\alpha$ & $0.48 \pm 0.05$ & $0.55 \pm 0.08$ & $0.425 \pm 0.008$ \\
$b$ & $-3.5 \pm 0.3$ & $-4.1 \pm 0.6$ & $-3.10 \pm 0.07$ \\
\hline & late & & late field \\
\hline$\alpha$ & $0.4 \pm 0.2$ & & $0.278 \pm 0.005$ \\
$b$ & $-3 \pm 1$ & & $-1.79 \pm 0.04$ \\
\hline & C-K07-I & & \\
\hline & early non-BCGs & $B C G s$ & early field \\
\hline$\alpha$ & $0.41 \pm 0.02$ & $0.55 \pm 0.08$ & $0.180 \pm 0.003$ \\
$b$ & $-2.9 \pm 0.2$ & $-4.2 \pm 0.7$ & $-0.91 \pm 0.03$ \\
\hline & late & & late field \\
\hline$\alpha$ & $0.40 \pm 0.08$ & & $0.30 \pm 0.01$ \\
$b$ & $-2.8 \pm 0.6$ & & $-1.88 \pm 0.08$ \\
\hline & & &
\end{tabular}

While the works mentioned above used de Vaucouleurs, isophotal or Sérsic models, we used Petrosian magnitudes and sizes. In addition, the sample selection causes variations in the value of the slopes; for example the use or not of spectroscopic data can introduce important differences in the results, as has been demonstrated by Coenda et al. (2006). Beyond the differences in the slopes, it should be noted that BCGs always follow a steeper relation than galaxies in control samples.

\subsection{Dynamical relations}

It is known that velocity dispersion and luminosity are well correlated for early-type galaxies $\left(L \propto \sigma^{\beta}\right)$, a relation known as Faber-Jackson (Faber \& Jackson 1976). Figure 7 shows the median values of $\log \sigma$ as function of $M_{r}^{0.1}$ for early-type galaxies in clusters (red lines), control sample (magenta lines) and $B C G_{\mathrm{s}}$ (black lines). Table 3 gives the parameters of the fits, where we can appreciate that there are no differences between fits corresponding to both cluster samples. Where we do see a clear difference is between galaxies in clusters (both $B C G_{\mathrm{s}}$ and non$\left.B C G_{\mathrm{s}}\right)$ and field galaxies, the latter being dynamically cooler (show lower mean velocity dispersions). The $\log \sigma-M_{r}^{0.1}$ also presents a departure from linearity for the brightest bin, where bright early type galaxies show a lower velocity dispersion than that expected from the linear relation that fits the lower magnitude bins. $B C G_{\mathrm{s}}$ show a $\log \sigma-M_{r}^{0.1}$ relation flatter than that of non-BCG galaxies. Nevertheless, the mean velocity dispersion that corresponds to the brightest luminosity bin of nonBCG galaxies is consistent with the values expected for $B C G_{\mathrm{s}}$. Therefore, the flattening of the $\log \sigma-M_{r}^{0.1}$ relation observed for $B C G_{\mathrm{s}}$ (see also Bernardi et al. 2007) could be a general property of early type galaxies regardless of its nature as the brightest cluster galaxy. Liu et al. (2007) and von der Linden et al. (2007) found that BCGs have a Faber-Jackson relation steeper than that of early-type galaxies. On the contrary, Bernardi et al. (2007) found a shallower relation for BCGs, consistent with our findings.

In Fig. 7 we found that early-type and $B C G_{\mathrm{s}}$ galaxies have, on average, the same velocity dispersions at a given luminosity. We also found that $B C G_{\mathrm{s}}$ have larger sizes than early type galaxies. Therefore, a different trend of dynamical masses as a function of luminosity between these two types of galaxies should be expected. In Fig. 8 we show the median value of $\log \left(r_{50} \sigma^{2}\right)$ as a function of the luminosity for early-types, $B G G_{\mathrm{s}}$ and controlsample galaxies. Again, the brightest bins show a departure from linearity and were excluded from the linear fits. For the three samples we plot a linear regression that confirm that $B C G_{\mathrm{s}}$ have, on average, higher dynamical masses than early-type galaxies of the same magnitude (the fitting parameters are given in Table 4). It should be noted that these differences are more significant for MaxBCG clusters (C-K07-I) than for X-ray clusters (C-P04-I). Field galaxies in control samples show lower dynamical masses than non-BCGs in clusters. 

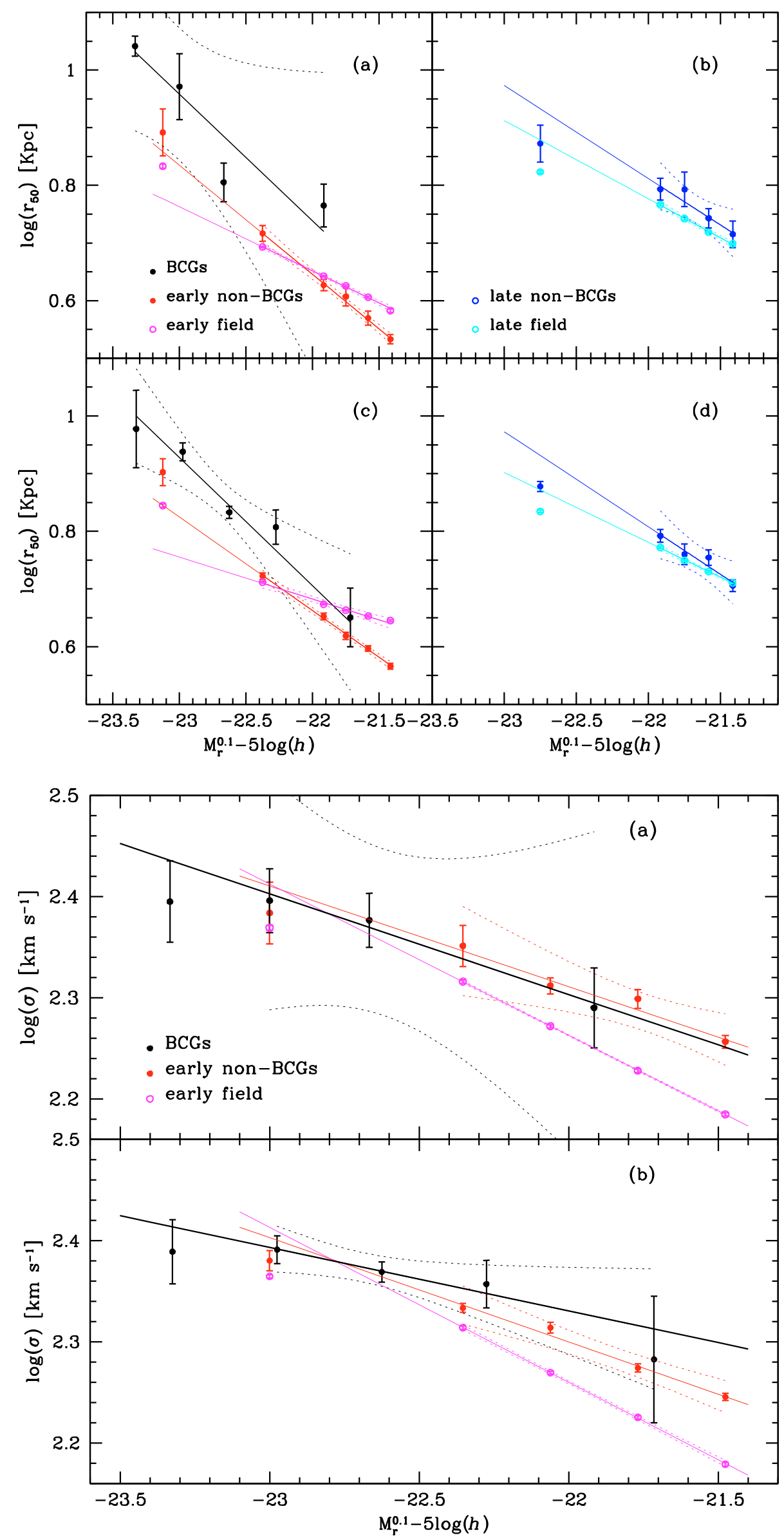

Fig. 6. The luminosity size relation: Median of $\log \left(r_{50}\right)$ versus $M_{r}^{0.1}$ for the C-P04-I sample (top panels) and the C-K07-I sample (bottom pan$e l s)$. Error bars were obtained by the bootstrap re-sampling technique. Dotted lines show the $95 \%$ confidence bands of the linear regression. The red and magenta lines show the linear regression of non- $B C G_{\mathrm{s}}$ early types in cluster and field respectively. The blue and cyan lines correspond to late types in cluster and field respectively. Black lines show the linear fits for $B C G_{\mathrm{s}}$.
Fig. 7. Faber-Jackson Relation: Linear fits of $\log (\sigma)$ versus $M_{r}^{0.1}$ for $n o n-B C G_{\mathrm{s}}$ early-types (red lines), $B C G_{\mathrm{s}}$ (black lines) and control samples (magenta lines). The panels correspond to the same cases as in Fig. 6. 


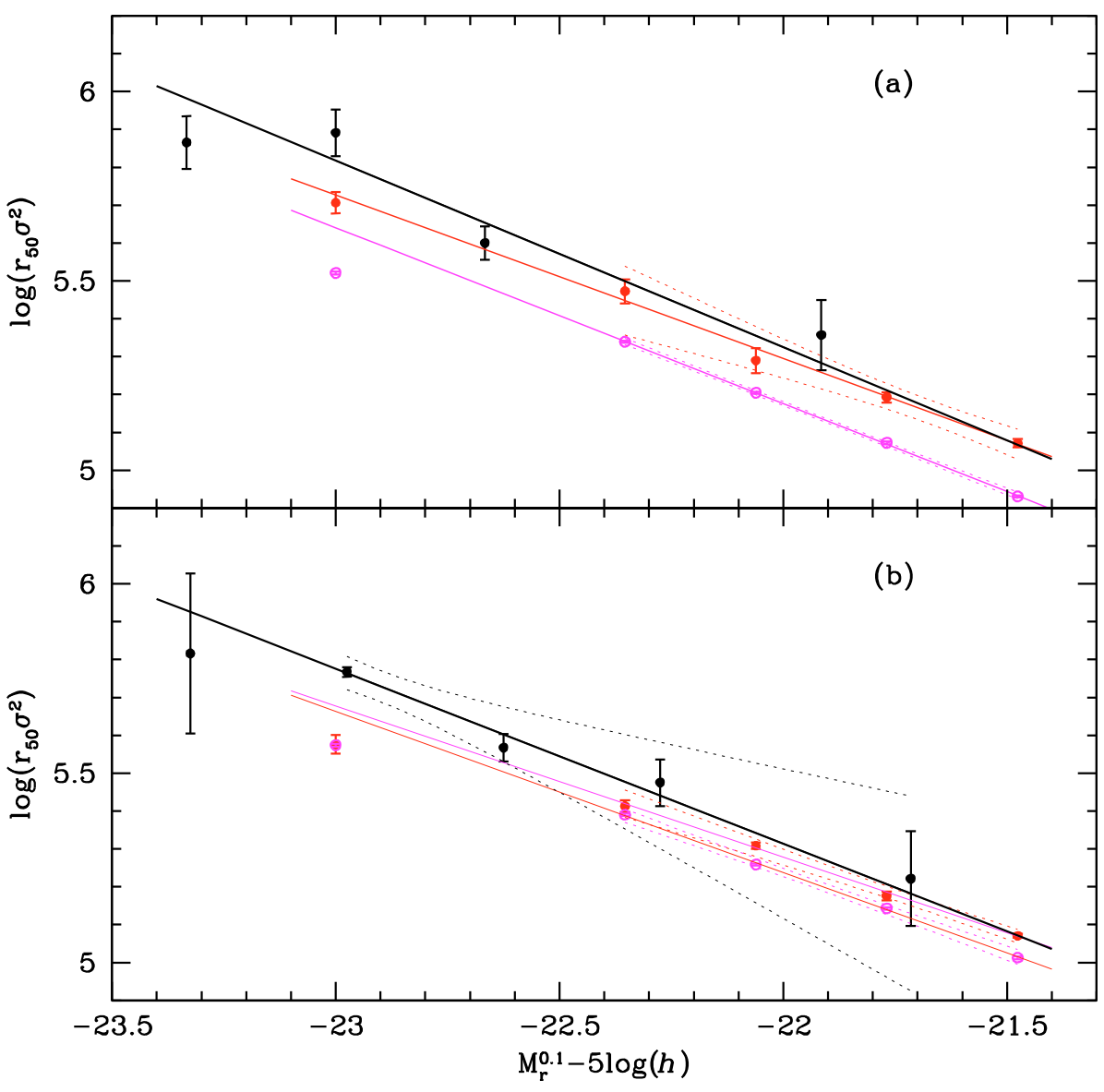

Fig. 8. Correlation between dynamical mass $r_{50 \sigma^{2}}$ and luminosity for early-types (red lines), $B C G_{\mathrm{s}}$ (black lines) and control samples (magenta lines), as in Fig. 7.
Table 3. Parameters of the fits $\log (\sigma)=-\frac{0.4}{\beta} M_{r}+b$, see Fig. 7 .

\begin{tabular}{llll}
\hline \hline & early non-BCGs & $B C G s$ & early field \\
\hline & C-P04-I & & F-P04-E \\
\hline$\beta$ & $4.0 \pm 0.8$ & $4 \pm 2$ & $2.67 \pm 0.05$ \\
$b$ & $-0.1 \pm 0.3$ & $0 \pm 1$ & $-1.03 \pm 0.06$ \\
\hline \hline & C-K07-I & & F-K07-E \\
\hline$\beta$ & $3.9 \pm 0.2$ & $7 \pm 3$ & $2.61 \pm 0.05$ \\
$b$ & $0.0 \pm 0.1$ & $1.0 \pm 0.7$ & $-1.11 \pm 0.06$ \\
\hline
\end{tabular}

Table 4. Parameters of the fits $\log \left(r_{50} \sigma^{2}\right)=a M_{r}+b$, see Fig. 8 .

\begin{tabular}{llll}
\hline \hline & early non-BCGs & BCGs & early field \\
\hline & C-P04-I & & F-P04-E \\
\hline$a$ & $-0.43 \pm 0.03$ & $-0.5 \pm 0.1$ & $-0.464 \pm 0.005$ \\
$b$ & $-4.2 \pm 0.7$ & $-6 \pm 2$ & $-5.0 \pm 0.1$ \\
\hline & C-K07-I & & F-K07-E \\
\hline$a$ & $-0.40 \pm 0.01$ & $-0.46 \pm 0.05$ & $-0.425 \pm 0.005$ \\
$b$ & $-3.5 \pm 0.3$ & $-5 \pm 1$ & $-4.1 \pm 0.1$ \\
\hline
\end{tabular}

\section{Galaxy segregation}

Using the C-P04-I and C-K07-I samples, Martínez et al. (2008) found that galaxy properties show a clear dependence on the clustocentric distance: a higher fraction of early type galaxies are found in the inner regions of clusters. In addition, they found that the $g-r$ colour is the property most predictive of the clustocentric distance of galaxies. Skibba et al. (2008) used SDSS galaxies with data from the Galaxy Zoo (Bamford et al. 2008) and found that for fixed morphology, the environmental dependence of colour remains strong. Blanton \& Berlind (2007) found that the relation between galaxy colors and the distance from the centre of groups has a residual relationship on the clustering of galaxies at small scales $\left(<300 h^{-1} \mathrm{Kpc}\right)$. In this work we are interested in the study of the segregation of several properties of bright galaxies in clusters as a function of $r / r_{200}$ and its dependence on the cluster identification techniques. Coenda et al. (2006) found that these type of analysis can produce different results depending on whether redshift-confirmed members or galaxies in projection are considered. Although we are only considering bright galaxies, our analysis is based on confirmed members in volume limited galaxy samples identified with two different criteria and galaxies extracted from the same galaxy catalogue. The characteristics of these samples make them suitable for an unbiased analysis of the properties of galaxies and its dependence on the cluster environment.

As we explained in Sect. 2.2, we have adopted several criteria to classify galaxies into morphological classes or types. We have used in our analysis the following parameters: the $g-r$ colour, the concentration index $C$, the eclass parameter and the Sersic index $n$. Although they are not independent, they are dominated by different physical properties of galaxies.

Figure 9 shows the fraction of early type galaxies as a function of the clustocentric radius. For both cluster samples a clear dependence of $r / r_{200}$ with $g-r, C$ and eclass is found (panels a) to c)). The correlation is up to $r / r_{200} \sim 1$, for larger clustocentric distances we do not see any tendency. For the Sersic in$\operatorname{dex} n$ (panel d)) we only observe a dependence for the C-K07-I sample. The comparison between the two cluster samples shows that the X-ray selected clusters always present a higher fraction of early type galaxies in the whole range of clustocentric distances. As it was pointed out previously, the X-ray sample has a slightly higher fraction of massive clusters than the MaxBCG 

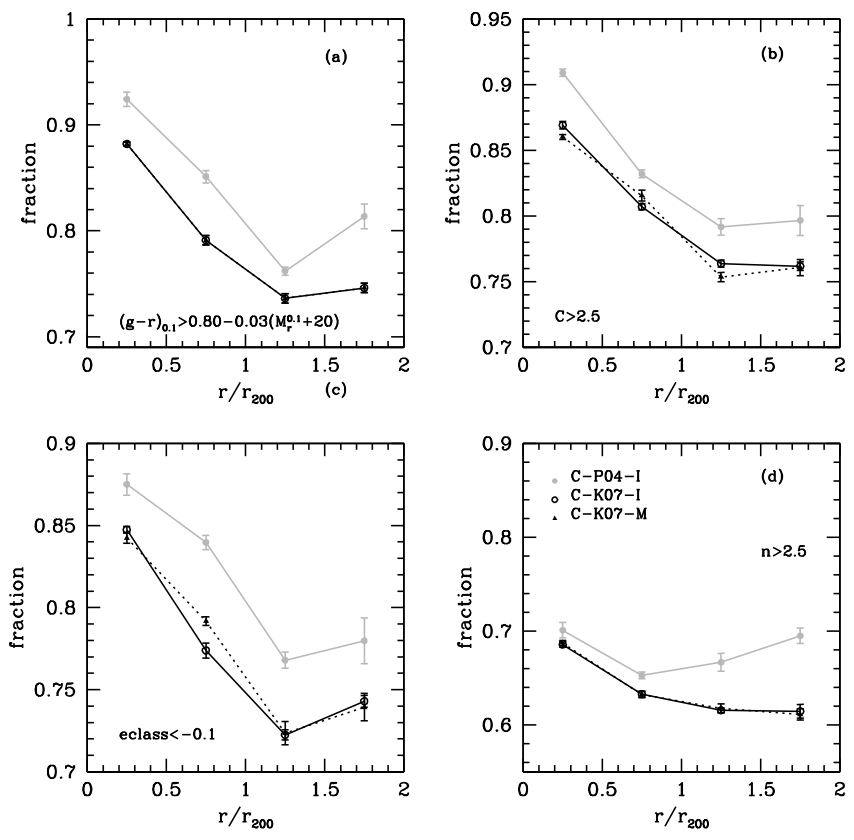

Fig. 9. Fraction of early type galaxies as a function of the normalized clustocentric radius. Early type galaxies are selected according to several parameters: $(g-r)^{0.1}$ in panel a), $C$ in panel b), eclass in panel c) and $n$ in panel d). The solid grey lines show the C-P04-I sample, whereas the solid black lines show the C-K07-I sample. The dotted black lines show the C-K07-M sample.

sample. In order to test if this difference in the mass distributions can be responsible for the higher fraction of early type galaxies in C-P04-I than in C-K07-I sample, we constructed a new subsample (C-K07-M) that consists of galaxies selected at random from C-K07-I having the same parent-cluster mass distribution as C-P04-I. As can be appreciated in Fig. 9, the fractions of early type galaxies as a function of $r / r_{200}$ for C-K07-M (dotted lines) are indistinguishable from those corresponding to the C-K07-I sample, indicating that our finding of a higher fraction of early type galaxies in X-ray than in MaxBCG clusters is independent of the dynamical mass distribution.

In panel (a) of Fig. 10 we show the fraction of galaxies brighter than $M_{r}^{0.1} \leq-22.5$ as a function of the normalized clustocentric distance. Analogously to the morphological segregation, we can see a clear dependence of this fraction for $r / r_{200} \lesssim 1$, in the sense that galaxies brighter $M_{r}^{0.1} \leq-22.5$ preferentially inhabit the cluster centres. This trend is stronger for the C-K07-I sample. Alternatively, in panel (b) we show the median values of $M_{r}^{0.1}$ as a function $r / r_{200}$. We observe the dependence between the median $M_{r}^{0.1}$ and $r / r_{200}$ for the C-K07-I sample. This effect is not observed for C-P04-I sample. The dispersion observed in mean values of $M_{r}^{0.1}$ (especially for C-P04-I sample) is due to the natural spread of the luminosity function.

As discussed in Sect. 3.1, the study of galaxy sizes and their relation to the luminosity helps to understand the galaxy formation and evolution. Here we study a possible dependence of galaxy size, $r_{50}$, as a function of $r / r_{200}$. Panel (a) in Fig. 11 shows the median value of $r_{50}$ versus the normalized clustocentric distance, for the C-P04-I sample (grey line) and for the C-K07-I sample (black line). For clustocentric distances larger than 0.8 , mean sizes of galaxies remain almost constant, being slightly larger for galaxies in X-ray clusters than in the MaxBCG sample, although the same effect is observed for field galaxies (horizontal dashed lines in Fig. 11). For smaller clustocentric distances, median sizes show different behaviour: for galaxies in the

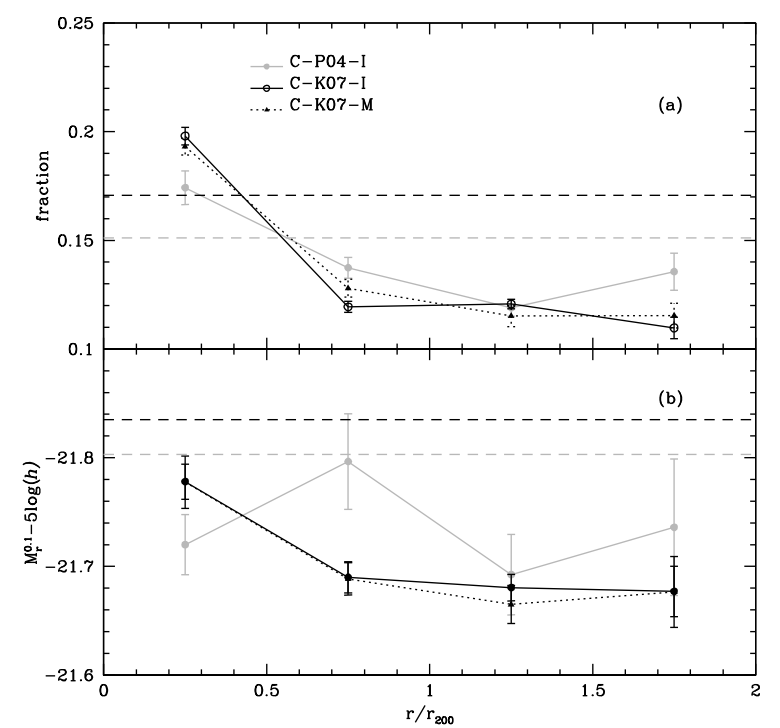

Fig. 10. Panel a) fraction of galaxies brighter than $M_{r}^{0.1} \leq-22.5$ as a function of $r / r_{200}$. The grey line shows the C-P04-I sample while the black line corresponds to the C-K07-I sample. The horizontal dashed lines show the median values of the corresponding field samples in the same colors as their cluster counterparts. Panel b) median values of $M_{r}^{0.1}$ versus $r / r_{200}$. The horizontal lines show the median values of the corresponding field samples.

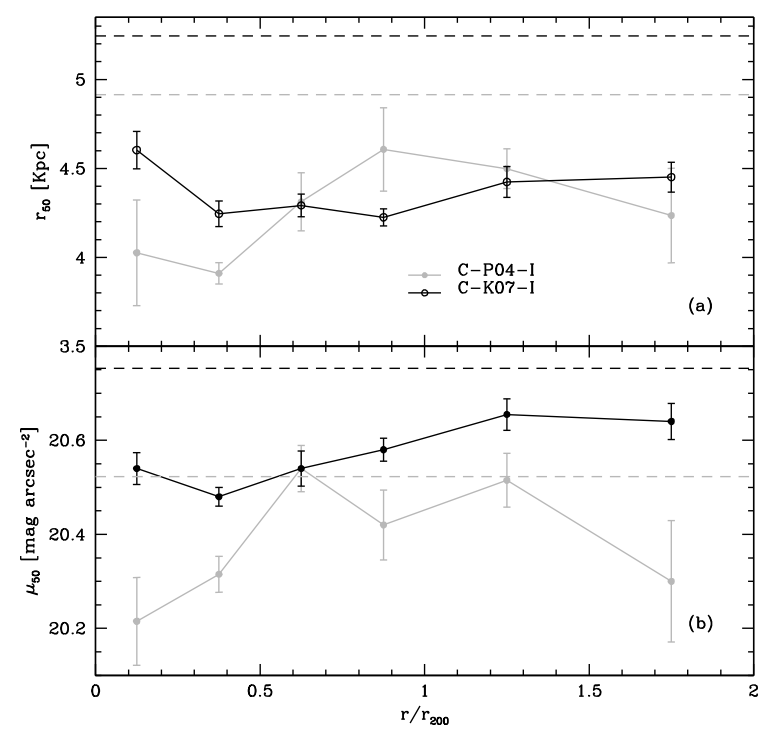

Fig. 11. Panel a) shows the median values of $r_{50}$ versus $r / r_{200}$ and panel b) the median values of $\mu_{50}$ versus $r / r_{200}$. The horizontal lines show the median values of the corresponding field samples, as in Fig. 10.

C-P04-I sample, median values of $r_{50}$ decrease as $r / r_{200}$ goes to zero, whereas the opposite behaviour is observed for galaxies in the C-K07-I sample. Panel (b) in Fig. 11 shows the median value of the $r$-band surface brightness $\mu_{50}$, computed inside $r_{50}$, as a function of $r / r_{200}$. For both cluster samples, $\mu_{50}$ decreases as $r / r_{200}$ goes to zero. Field galaxies have lower surface brightness (black horizontal line) than cluster members.

Figure 12 repeats the previous analysis using only early type galaxies. In the left panels we show this relation for the C-P04I sample while C-K07-I sample are shown in the right panels. We have also evaluated the influence of $B C G_{\mathrm{s}}$ in our results, repeating the analysis without $B C G_{\mathrm{s}}$ (dotted lines). Analogously to Fig. 11, the sizes of early type galaxies increase when $r / r_{200}$ decreases in the C-K07-I sample. Nevertheless, this tendency 

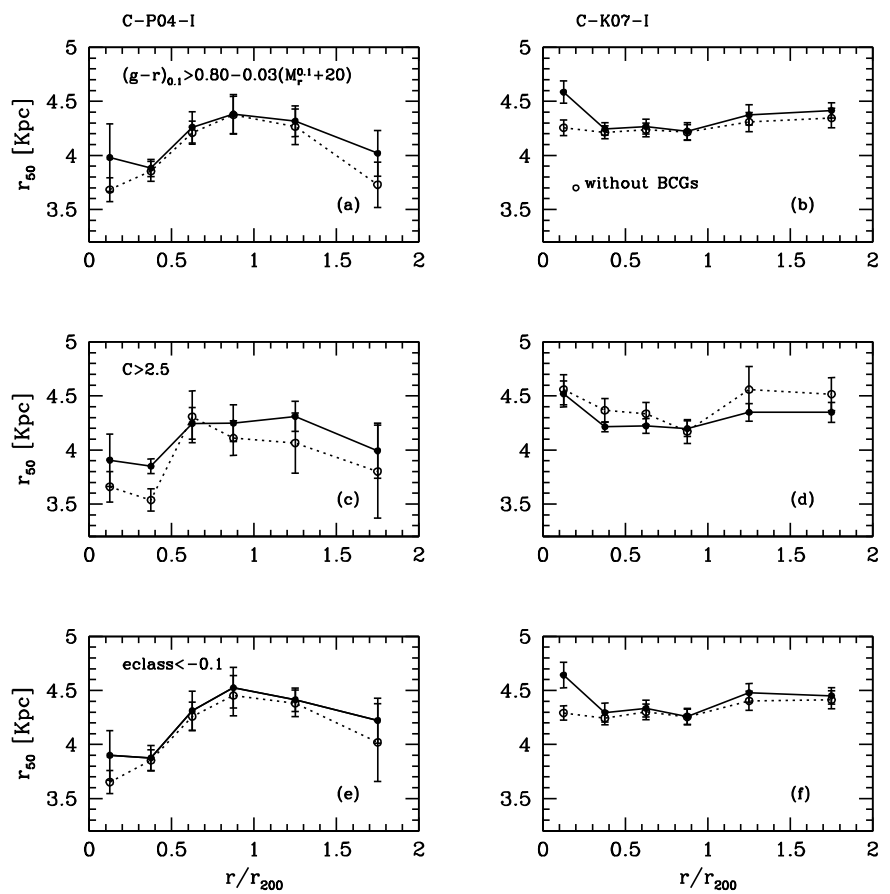

Fig. 12. Median values of $r_{50}$ as a function of $r / r_{200}$ for early-type galaxies. In the left panels we show this relation for the C-P04-I sample while C-K07-I clusters are shown on the right panels. Early type galaxies were selected according to several parameters: $(g-r)^{0.1}$ in a) and b), $C$ in c) and d), and eclass in e) and f). Dotted lines show the same relation but excluding the $B C G_{\mathrm{s}}$.

disappears when $B C G_{\mathrm{s}}$ are excluded. On the other hand, the median values of $r_{50}$ decrease as $r / r_{200}$ goes to zero for the C-P04-I sample; this effect is stronger when $B C G_{\mathrm{s}}$ are excluded. These results clearly show an important difference between clusters identified with different criteria. As can be appreciated in Fig. 3, a higher fraction of early type galaxies is observed for the X-ray selected clusters. Consequently, the different behaviour observed in Fig. 12, could be the result of the differences in the population of early type galaxies between the two samples. For instance, a higher fraction of elliptical galaxies in the early type population of the X-ray selected clusters with respect to the MaxBCG sample could produce a different behaviour in the mean sizes as a function of $r / r_{200}$. In order to test this possibility, we repeated the analysis of Fig. 12 selecting early type galaxies with $C>3.1$. This new threshold includes a third of the early type galaxies previously analyzed, and it will mostly select elliptical galaxies. For this sub-sample of early type galaxies, we find the same trend observed in Fig. 12, confirming that there is a dependence of the mean sizes of early type galaxies on the cluster selection criterion. Based on a sample of 228 elliptical galaxies in 24 clusters, Cypriano et al. (2006) found that elliptical galaxies in the inner regions of clusters are typically 5\% smaller than those in the outer regions. These authors interpreted these results in terms of the tidal stripping of stars that lead to changes in the whole structure of galaxies in the central regions of clusters.

Figure 13 is the analogous of Fig. 12 for late-type galaxies. Although galaxies in X-ray selected clusters show a tendency on average $r_{50}$ to be smaller towards the cluster centers, this behaviour is not statistically significant.

\section{Discussion and conclusions}

We have compiled two catalogues of massive clusters of galaxies based on samples that make use of different identification

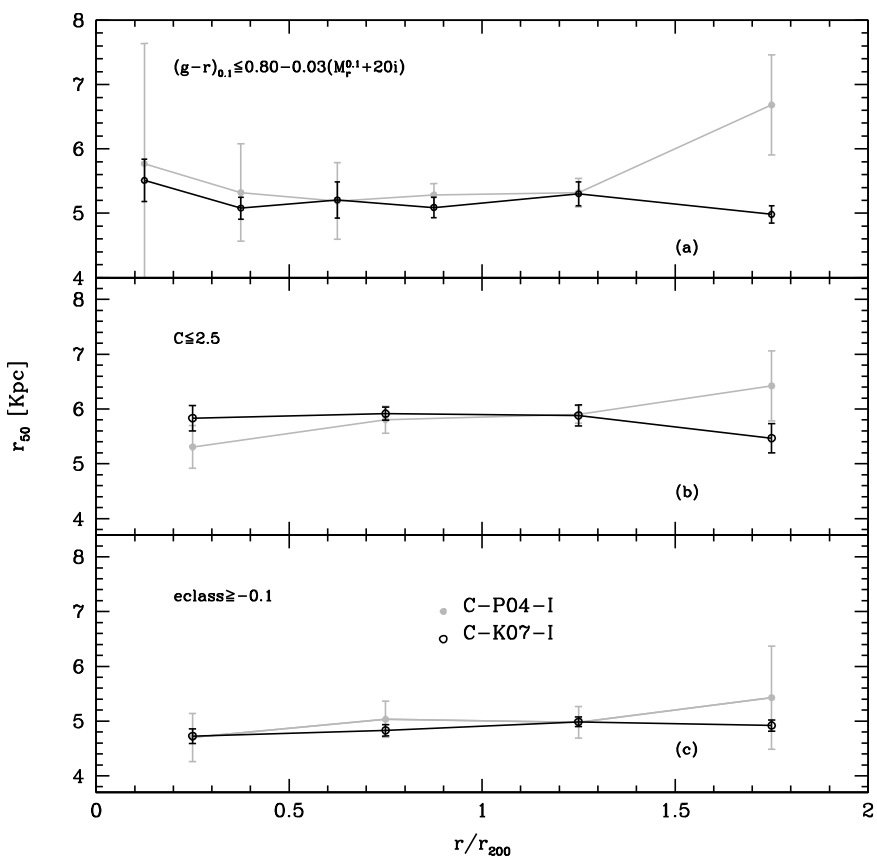

Fig. 13. Median values of $r_{50}$ as a function of $r / r_{200}$ for late-type galaxies, analogously to Fig. 12.

techniques: the P04 sample that comprises bright X-ray galaxy clusters and the K07 sample, constructed according to the properties of early-type galaxies. We selected sub-samples of galaxy clusters in the redshift range $0.05<z<0.14$. For both samples, member galaxies are brighter that $M_{r}^{0.1}=-21.3$ and were identified from a spectroscopic volume-limited sample of the DR5SDSS. In order to select a fair sample of clusters of galaxies we use the fof algorithm and an eyeball examination of each overdensity identified by fof. Throughout the visual inspection, we classified the clusters based on their substructure. We only consider in our analysis regular clusters and we exclude systems that have two or more close substructures of similar size in the plane of the sky and/or in the redshift distribution. In addition, we have determined new positions in the plane of the sky and in the line of sight. We found that for $\sim 40 \%$ of the clusters, the angular position given by fof is better than the original value, whereas for $\sim 17 \%$ of the clusters, the redshift given by fof is a better match to the observed mean redshift distribution than the original value. Our final sample comprises 49 X-ray selected clusters with 786 galaxies and 209 MaxBCG clusters with 3041 galaxies. We have determined the following physical properties of clusters: the line-of-sight velocity dispersion $\sigma$, the virial radius and mass and $r_{200}$.

We have analyzed different scaling relations between photometric and dynamical parameters. Bright galaxies were separated in early and late types according to several criteria. We have also studed the segregation of galaxies analysing different properties as a function of the normalized clustocentric distances. The main results are:

- Galaxies in X-ray and MaxBCG selected clusters show similar size-luminosity relations.

- We found that each galaxy type has a different luminositysize relation. This implies a different mass-luminosity relation and/or a different star formation history.

- For each luminosity bin, late type galaxies in the field have sizes smaller than their cluster counterpart. 
- The same effect is observed for early type galaxies, although the differences are smaller. The stronger effect corresponds to galaxies in MaxBCG clusters.

- At fixed luminosity, $B C G_{\mathrm{s}}$ tend to be larger and show a steeper $\log \left(r_{50}\right)-M_{r}^{0.1}$ relation than non-BCG early type galaxies in clusters.

- Non-BCGs early type galaxies in clusters and in the field show the known curvature in the size-luminosity relation.

- The Faber-Jackson relation for early-type galaxies in clusters is the same in X-ray and MaxBCG clusters.

- We found a clear difference between galaxies in clusters (both $B C G_{\mathrm{s}}$ and non-BCG) and in the field, showing the later lower values of the velocity dispersion at fixed luminosity.

- The $\log \sigma-M_{r}^{0.1}$ relation presents, at the bright end, a departure from linearity. Bright early type galaxies show a lower velocity dispersion than the expected from the linear relation at lower magnitudes.

- $B C G_{\mathrm{s}}$ show a flatter $\log \sigma-M_{r}^{0.1}$ relation than non-BCG galaxies. Nevertheless, the velocity dispersion of the brightest non-BCG galaxies are consistent with the values expected for $B C G_{\mathrm{s}}$.

- At fixed luminosity, $B C G_{\mathrm{s}}$ have, on average, higher dynamical masses than early-type galaxies in clusters. The difference is more significant for MaxBCG clusters than for X-ray selected clusters.

- At fixed luminosity, field early type galaxies in the control samples show lower dynamical masses than non-BCG early type galaxies in clusters.

- Using several criteria to classify galaxies into morphological classes, we found the well know morphological segregation for both samples of clusters. The correlation is up to $r / r_{200} \sim$ 1; for larger clustocentric distances we do not see any trend.

- For the whole range of clustocentric distances, X-ray selected clusters have a higher fraction of early type galaxies than MaxBCG clusters.

- Within $r / r_{200} \sim 1$ we found a dependence of the fraction of galaxies brighter than $M_{r}^{0.1} \leq-22.5$ as a function of the normalized clustocentric distance. Bright galaxies preferentially inhabit the cluster centers. This tendency is stronger for MaxBCG clusters.

- For clustocentric distances larger than 0.8, mean sizes of galaxies remain almost constant, being slightly larger for galaxies in X-ray selected clusters than in the MaxBCG sample.

- For $r / r_{200} \leq 0.8$, median sizes depend on the cluster sample. For galaxies in $\mathrm{X}$-ray selected clusters, median values of $r_{50}$ decrease as $r / r_{200}$ goes to zero, whereas the opposite is observed for galaxies in the MaxBCG clusters.

- For MaxBCG clusters, the sizes of early type galaxies increase as $r / r_{200}$ decreases. The trend disappears when $B C G_{\mathrm{S}}$ are excluded. On the contrary, the median value of $r_{50}$ decrease as $r / r_{200}$ goes to zero for X-ray selected clusters. The effect is stronger when $B C G_{\mathrm{s}}$ are excluded.

Early type galaxies in the field are lower, have lower velocity dispersion and have smaller virial masses than their cluster counterpart. On the other hand, $B C G_{\mathrm{s}}$ are larger and have higher values of the virial masses than non-BCG in clusters, although the velocity dispersions are comparable. These differences in the properties of early type galaxies in the field, in clusters and with $B C G_{\mathrm{s}}$ are consistent with the scenario where the environment plays a fundamental rol in the formation histories of galaxies. These results suggest three different formation scenarios, $B C G_{\mathrm{s}}$ being the galaxies less affected by dissipation. Dry mergers have no energy loss mechanism, therefore the formed galaxies are less centrally contracted with optical sizes larger than those of galaxies that have undergone a dissipative merger (Kormendy 1989; Nipoti et al. 2003; Bernardi et al. 2007). Weinmann et al. (2009) and Guo et al. (2009) analyzed the size-luminosity relation of galaxies in groups, where the environmental effects are supposed to be different to those expected in clusters. These authors found the same size-luminosity relation for both central and satellite early type galaxies. The different results between galaxies in groups and clusters also support the idea that the size-luminosity relation depends on the environment.

At the same luminosity, late-type galaxies in clusters have larger sizes, and therefore lower surface brightness than their field counterparts (control sample). This is in agreement with the scenario of strangulation (Larson et al. 1980), where the gas in the halo is stripped, suppressing the supply of cold gas and therefore affecting the star formation rate and the galaxy luminosity.

The X-ray selected sample C-P04-I is made of bright X-ray galaxy clusters, whereas the C-K07-I sample was constructed according to the properties of early-type galaxies. Several clusters in the C-K07-I sample should be X-ray emitters, although they would be, on average, less X-ray luminous than C-P04I clusters. The fact that X-ray selected clusters show a higher fraction of early type galaxies whose sizes tend to decrease towards the cluster center, effect that is not observed for galaxies in MaxBCG clusters, can be interpreted as possible evidence that the hot intracluster medium is also playing a role in the evolution of early type galaxies. Alternatively, the observed effect could be caused by differences in the clusters masses. Even though the effects mentioned above are also present for sub-samples of CP04-I and C-K07-I with the same virial mass distribution, due to the usual uncertainties in the computation of these masses, we cannot discard differences in the actual mass distributions. If this is the case, and C-P04-I clusters are on average more massive than C-K07-I, decreases in the mean sizes of early type galaxies in X-ray selected clusters could be the result of tidal stripping that can produce changes in the structure of galaxies (see the results from numerical simulations of Aguilar \& White (1986) and observational evidences from Trujillo et al. (2002) and Cypriano et al. (2006)). This difference between X-ray and MaxBCG selected clusters is an unexpected result that should be explored in more detail.

Acknowledgements. This work has been partially supported with grants from Consejo Nacional de Investigaciones Científicas y Técnicas de la República Argentina (CONICET) and Secretaría de Ciencia y Tecnología de la Universidad de Córdoba. We kindly thank Dr. Héctor J. Martínez for his helpful comments on the manuscript, which contributed to improve the present paper. VC acknowledges the guidance of Dr. Ariel Zandivarez in the construction of the cluster sample.

\section{References}

Adelman-McCarthy, J. K., Agüeros, M. A., Allam, S. S., et al. 2007, ApJS, 172, 634

Adelman-McCarthy, J. K., Agüeros, M. A., Allam, S. S., et al. 2008, ApJS, 175, 297

Aguilar, L. A., \& White, S. D. M. 1986, ApJ, 307, 97

Andredakis, Y. C., Peletier, R. F., \& Balcells, M. 1995, MNRAS, 275, 874

Bahcall, N. A. 1973, ApJ, 183, 783

Ball, N. M., Loveday, J., \& Brunner, R. J. 2008, MNRAS, 383, 907

Bamford, S. P., Rojas, A. L., Nichol, R. C., et al. 2008, MNRAS, 391, 607

Beers, T. C., Flynn, K., \& Gebhardt, K. 1990, AJ, 100, 32

Bernardi, M. 2009, MNRAS, 510

Bernardi, M., Sheth, R. K., Annis, J., et al. 2003, AJ, 125, 1866

Bernardi, M., Sheth, R. K., Nichol, R. C., Schneider, D. P., \& Brinkmann, J. 2005, AJ, 129, 61 
Bernardi, M., Hyde, J. B., Sheth, R. K., Miller, C. J., \& Nichol, R. C. 2007, AJ, 133, 1741

Bildfell, C., Hoekstra, H., Babul, A., \& Mahdavi, A. 2008, MNRAS, 934

Biviano, A., Katgert, P., Thomas, T., \& Adami, C. 2002, A\&A, 387, 8

Blanton, M. R., \& Berlind, A. A. 2007, ApJ, 664, 791

Blanton, M. R., Brinkmann, J., Csabai, I., et al. 2003, AJ, 125, 2348

Blanton, M. R., Eisenstein, D., Hogg, D. W., Schlegel, D. J., \& Brinkmann, J. 2005a, ApJ, 629, 143

Blanton, M. R., Schlegel, D. J., Strauss, M. A., et al. 2005b, AJ, 129, 2562

Carlberg, R. G., Yee, H. K. C., \& Ellingson, E. 1997, ApJ, 478, 462

Coenda, V., Donzelli, C. J., Muriel, H., et al. 2005, AJ, 129, 1237

Coenda, V., Muriel, H., Donzelli, C. J., et al. 2006, AJ, 131, 1989

Cross, N. J. G., Bouwens, R. J., Benítez, N., et al. 2004, AJ, 128, 1990

Cypriano, E. S., Sodré, L. J., Campusano, L. E., Dale, D. A., \& Hardy, E. 2006, AJ, 131, 2417

de Jong, R. S., \& Lacey, C. 2000, ApJ, 545, 781

Díaz, E., Zandivarez, A., Merchán, M. E., \& Muriel, H. 2005, ApJ, 629, 158

Djorgovski, S., \& Davis, M. 1987, ApJ, 313, 59

Domínguez, M., Muriel, H., \& Lambas, D. G. 2001, AJ, 121, 1266

Dressler, A. 1980, ApJS, 42, 565

Dressler, A., Lynden-Bell, D., Burstein, D., et al. 1987, ApJ, 313, 42

Dressler, A., Oemler, A. J., Couch, W. J., et al. 1997, ApJ, 490, 577

Driver, S. P., Odewahn, S. C., Echevarria, L., et al. 2003, AJ, 126, 2662

Driver, S. P., Allen, P. D., Graham, A. W., et al. 2006, MNRAS, 368, 414

Faber, S. M., \& Jackson, R. E. 1976, ApJ, 204, 668

Ferguson, H. C., Dickinson, M., Giavalisco, M., et al. 2004, ApJ, 600, L107

Folkes, S., Ronen, S., Price, I., et al. 1999, MNRAS, 308, 459

Girardi, M., Biviano, A., Giuricin, G., Mardirossian, F., \& Mezzetti, M. 1993, ApJ, 404, 38

Goto, T., Sekiguchi, M., Nichol, R. C., et al. 2002, AJ, 123, 1807

Guo, Y., McIntosh, D. H., Mo, H. J., et al. 2009, ArXiv e-prints

Huchra, J. P., \& Geller, M. J. 1982, ApJ, 257, 423

Hyde, J. B., \& Bernardi, M. 2009, MNRAS, 349

Kashikawa, N., Sekiguchi, M., Doi, M., et al. 1998, ApJ, 500, 750

Koester, B. P., McKay, T. A., Annis, J., et al. 2007a, ApJ, 660, 239

Koester, B. P., McKay, T. A., Annis, J., et al. 2007b, ApJ, 660, 221

Kormendy, J. 1977, ApJ, 218, 333

Kormendy, J. 1989, ApJ, 342, L63

La Barbera, F., Merluzzi, P., Busarello, G., Massarotti, M., \& Mercurio, A. 2004, A\&A, 425, 797

Larson, R. B., Tinsley, B. M., \& Caldwell, C. N. 1980, ApJ, 237, 692

Lauer, T. R., Faber, S. M., Richstone, D., et al. 2007, ApJ, 662, 808

Liu, F. S., Xia, X. Y., Mao, S., Wu, H., \& Deng, Z. G. 2007, ArXiv e-prints, 712

Lobo, C., Biviano, A., Durret, F., et al. 1997, A\&A, 317, 385

Loveday, J., Peterson, B. A., Efstathiou, G., \& Maddox, S. J. 1992, ApJ, 390, 338

Madgwick, D. S., Lahav, O., Baldry, I. K., et al. 2002, MNRAS, 333, 133
Malumuth, E. M., \& Kirshner, R. P. 1981, ApJ, 251, 508

Malumuth, E. M., \& Kirshner, R. P. 1985, ApJ, 291, 8

Martínez, H. J., Coenda, V., \& Muriel, H. 2008, [arXiv:0809. 1585]

McIntosh, D. H., Bell, E. F., Rix, H.-W., et al. 2005, ApJ, 632, 191

Melnick, J., \& Sargent, W. L. W. 1977, ApJ, 215, 401

Merchán, M. E., \& Zandivarez, A. 2005, ApJ, 630, 759

Miller, C. J., Nichol, R. C., Reichart, D., et al. 2005, AJ, 130, 968

Möllenhoff, C., \& Heidt, J. 2001, A\&A, 368, 16

Nipoti, C., Londrillo, P., \& Ciotti, L. 2003, MNRAS, 342, 501

Noonan, T. 1961, PASP, 73, 212

Oegerle, W. R., \& Hoessel, J. G. 1991, ApJ, 375, 15

Oemler, A. J. 1974, ApJ, 194, 1

Peng, C. Y., Ho, L. C., Impey, C. D., \& Rix, H.-W. 2002, AJ, 124, 266

Petrosian, V. 1976, ApJ, 209, L1

Popesso, P., Böhringer, H., Brinkmann, J., Voges, W., \& York, D. G. 2004, A\&A, 423, 449

Pracy, M. B., Driver, S. P., De Propris, R., Couch, W. J., \& Nulsen, P. E. J. 2005, MNRAS, 364, 1147

Rood, H. J., \& Turnrose, B. E. 1968, ApJ, 152, 1057

Sandage, A., \& Perelmuter, J.-M. 1990, ApJ, 361, 1

Sandage, A., \& Visvanathan, N. 1978a, ApJ, 225, 742

Sandage, A., \& Visvanathan, N. 1978b, ApJ, 223, 707

Schechter, P. 1976, ApJ, 203, 297

Schlegel, D. J., Finkbeiner, D. P., \& Davis, M. 1998, ApJ, 500, 525

Sérsic, J. L. 1963, Boletín de la Asociación Argentina de Astronomía, 6, 41

Sérsic, J. L. 1968, Atlas de galaxias australes (Córdoba, Argentina: Observatorio Astronómico)

Shen, S., Mo, H. J., White, S. D. M., et al. 2003, MNRAS, 343, 978

Shimasaku, K., Fukugita, M., Doi, M., et al. 2001, AJ, 122, 1238

Skibba, R. A., Bamford, S. P., Nichol, R. C., et al. 2008, ArXiv e-prints

Strateva, I., Ivezić, Ž., Knapp, G. R., et al. 2001, AJ, 122, 1861

Strauss, M. A., Weinberg, D. H., Lupton, R. H., et al. 2002, AJ, 124, 1810

Syer, D., Mao, S., \& Mo, H. J. 1999, MNRAS, 305, 357

Trujillo, I., Aguerri, J. A. L., Gutiérrez, C. M., Caon, N., \& Cepa, J. 2002, ApJ, 573, L9

Trujillo, I., Förster Schreiber, N. M., Rudnick, G., et al. 2006, ApJ, 650, 18

Tully, R. B., \& Fisher, J. R. 1977, A\&A, 54, 661

von der Linden, A., Best, P. N., Kauffmann, G., \& White, S. D. M. 2007, MNRAS, 379, 867

Weinmann, S. M., Kauffmann, G., van den Bosch, F. C., et al. 2009, MNRAS, 394, 1213

Whitmore, B. C., Gilmore, D. M., \& Jones, C. 1993, ApJ, 407, 489

Yang, X., Mo, H. J., van den Bosch, F. C., et al. 2007, ApJ, 671, 153

Yepes, G., Dominguez-Tenreiro, R., \& del Pozo-Sanz, R. 1991, ApJ, 373, 336

Yip, C. W., Connolly, A. J., Szalay, A. S., et al. 2004, AJ, 128, 585

York, D. G., Anderson, Jr., J. E., Anderson, S. F., et al. 2000, AJ, 120, 1579

Ziegler, B. L., Saglia, R. P., Bender, R., et al. 1999, A\&A, 346, 13 
V. Coenda and H. Muriel: Galaxy properties in clusters, Online Material p 1

Table A.1. C-P04-I cluster sample.

\begin{tabular}{|c|c|c|c|c|c|c|}
\hline $\begin{array}{l}\alpha(\mathrm{J} 2000.00) \\
{[\mathrm{h} \mathrm{m} \mathrm{s}]}\end{array}$ & $\begin{array}{l}\delta(\mathrm{J} 2000.00) \\
{\left[{ }^{\circ},{ }^{\prime}\right]}\end{array}$ & $z$ & $\begin{array}{c}\sigma \\
{\left[\mathrm{km} \mathrm{s}^{-1}\right]}\end{array}$ & $\begin{array}{c}M_{\mathrm{vir}} \\
{\left[h^{-1} M_{\odot}\right]}\end{array}$ & $\begin{array}{c}R_{\text {vir }} \\
{\left[h^{-1} \mathrm{Mpc}\right]}\end{array}$ & $\begin{array}{c}R_{200} \\
{\left[h^{-1} \mathrm{Mpc}\right]}\end{array}$ \\
\hline 04150.1 & -9186.8 & 0.056 & 698.82 & $0.558 \times 10^{15}$ & 1.638 & 1.729 \\
\hline 11937.7 & +145335.2 & 0.129 & 778.90 & $0.502 \times 10^{15}$ & 1.185 & 1.927 \\
\hline 73625.0 & +392558.4 & 0.117 & 605.19 & $0.482 \times 10^{15}$ & 1.887 & 1.497 \\
\hline 75319.0 & +292226.8 & 0.061 & 689.22 & $0.606 \times 10^{15}$ & 1.829 & 1.705 \\
\hline 8940.2 & +345534.3 & 0.082 & 528.46 & $0.323 \times 10^{15}$ & 1.656 & 1.308 \\
\hline 81022.6 & +42160.8 & 0.064 & 548.75 & $0.292 \times 10^{15}$ & 1.391 & 1.358 \\
\hline 82210.0 & +47558.2 & 0.130 & 674.55 & $0.671 \times 10^{15}$ & 2.114 & 1.669 \\
\hline 82527.6 & +47710.6 & 0.126 & 792.06 & $0.952 \times 10^{15}$ & 2.175 & 1.960 \\
\hline 8286.7 & +444548.2 & 0.145 & 577.57 & $0.187 \times 10^{15}$ & 0.803 & 1.429 \\
\hline 84529.0 & +443428.2 & 0.054 & 331.69 & $0.545 \times 10^{14}$ & 0.710 & 0.821 \\
\hline 9139.2 & +474326.4 & 0.052 & 408.98 & $0.120 \times 10^{15}$ & 1.032 & 1.012 \\
\hline 95341.5 & +14242.5 & 0.098 & 403.63 & $0.165 \times 10^{15}$ & 1.454 & 0.999 \\
\hline 102341.1 & +4985.6 & 0.144 & 618.10 & $0.532 \times 10^{15}$ & 1.997 & 1.529 \\
\hline 10545.5 & +545050.6 & 0.072 & 448.67 & $0.192 \times 10^{15}$ & 1.368 & 1.110 \\
\hline 105826.3 & +564731.9 & 0.136 & 597.87 & $0.332 \times 10^{15}$ & 1.330 & 1.479 \\
\hline $\begin{array}{lll}11 & 1322.7\end{array}$ & +23232.6 & 0.075 & 431.97 & $0.170 \times 10^{15}$ & 1.304 & 1.069 \\
\hline $11 \quad 1532.2$ & +54265.6 & 0.070 & 628.67 & $0.334 \times 10^{15}$ & 1.213 & 1.556 \\
\hline 112136.2 & +48350.0 & 0.112 & 728.02 & $0.541 \times 10^{15}$ & 1.462 & 1.801 \\
\hline 113317.3 & +662245.5 & 0.115 & 847.57 & $0.934 \times 10^{15}$ & 1.865 & 2.097 \\
\hline 11448.2 & +54522.7 & 0.103 & 586.83 & $0.370 \times 10^{15}$ & 1.541 & 1.452 \\
\hline 114440.8 & +672440.0 & 0.117 & 453.55 & $0.221 \times 10^{15}$ & 1.542 & 1.122 \\
\hline 12024.5 & +31951.6 & 0.133 & 858.09 & $0.119 \times 10^{16}$ & 2.310 & 2.123 \\
\hline 121740.8 & +33941.0 & 0.077 & 922.14 & $0.113 \times 10^{16}$ & 1.903 & 2.282 \\
\hline 125841.1 & -14524.8 & 0.084 & 740.78 & $0.769 \times 10^{15}$ & 2.010 & 1.833 \\
\hline 13250.7 & -23022.3 & 0.083 & 681.06 & $0.534 \times 10^{15}$ & 1.650 & 1.685 \\
\hline 13356.5 & +67313.7 & 0.106 & 659.69 & $0.358 \times 10^{15}$ & 1.179 & 1.632 \\
\hline 13917.0 & -13645.4 & 0.083 & 519.51 & $0.238 \times 10^{15}$ & 1.264 & 1.285 \\
\hline 132617.8 & +0 1332.5 & 0.082 & 501.04 & $0.242 \times 10^{15}$ & 1.382 & 1.240 \\
\hline 133049.9 & -15222.1 & 0.087 & 659.17 & $0.480 \times 10^{15}$ & 1.585 & 1.631 \\
\hline 133238.9 & +54199.5 & 0.101 & 811.68 & $0.927 \times 10^{15}$ & 2.018 & 2.008 \\
\hline 13366.5 & +591226.6 & 0.071 & 934.40 & $0.123 \times 10^{16}$ & 2.017 & 2.312 \\
\hline $1342 \quad 28.3$ & +21445.2 & 0.077 & 752.26 & $0.686 \times 10^{15}$ & 1.738 & 1.861 \\
\hline 13530.8 & +5921.2 & 0.079 & 755.36 & $0.579 \times 10^{15}$ & 1.454 & 1.869 \\
\hline 141447.4 & -02356.8 & 0.140 & 612.45 & $0.448 \times 10^{15}$ & 1.712 & 1.515 \\
\hline 142440.4 & +24446.7 & 0.055 & 558.59 & $0.208 \times 10^{15}$ & 0.954 & 1.382 \\
\hline 142522.9 & +631122.6 & 0.139 & 1052.31 & $0.198 \times 10^{16}$ & 2.557 & 2.604 \\
\hline 151251.1 & -12847.3 & 0.122 & 812.48 & $0.895 \times 10^{15}$ & 1.943 & 2.010 \\
\hline 151634.0 & -0 5655.7 & 0.118 & 539.58 & $0.385 \times 10^{15}$ & 1.897 & 1.335 \\
\hline $\begin{array}{lll}15 & 29 & 12.1\end{array}$ & +525039.8 & 0.074 & 694.40 & $0.522 \times 10^{15}$ & 1.553 & 1.718 \\
\hline 16122.1 & +53 5419.1 & 0.106 & 491.24 & $0.284 \times 10^{15}$ & 1.686 & 1.216 \\
\hline $1611 \quad 17.7$ & +365738.2 & 0.067 & 529.83 & $0.275 \times 10^{15}$ & 1.406 & 1.311 \\
\hline 165620.3 & +391659.9 & 0.062 & 440.23 & $0.140 \times 10^{15}$ & 1.034 & 1.089 \\
\hline 165945.4 & +323658.0 & 0.101 & 512.65 & $0.257 \times 10^{15}$ & 1.403 & 1.268 \\
\hline 17242.6 & +34340.7 & 0.099 & 1060.16 & $0.161 \times 10^{16}$ & 2.059 & 2.623 \\
\hline 171247.6 & +64347.5 & 0.080 & 1037.59 & $0.167 \times 10^{16}$ & 2.223 & 2.567 \\
\hline $17 \quad 189.9$ & +563959.0 & 0.113 & 676.01 & $0.565 \times 10^{15}$ & 1.772 & 1.673 \\
\hline 212456.3 & -65647.4 & 0.118 & 809.90 & $0.102 \times 10^{16}$ & 2.224 & 2.004 \\
\hline 215725.8 & -74740.6 & 0.058 & 649.63 & $0.498 \times 10^{15}$ & 1.692 & 1.607 \\
\hline $2216 \quad 15.5$ & -92023.6 & 0.084 & 488.35 & $0.218 \times 10^{15}$ & 1.311 & 1.208 \\
\hline
\end{tabular}


V. Coenda and H. Muriel: Galaxy properties in clusters, Online Material p 2

Table A.2. Analogous to Table A.1, for C-K07-I cluster sample.

\begin{tabular}{|c|c|c|c|c|c|c|}
\hline $\begin{array}{l}\alpha(\mathrm{J} 2000.00) \\
{[\mathrm{h} \mathrm{m} \mathrm{s}]}\end{array}$ & $\begin{array}{l}\delta(\mathrm{J} 2000.00) \\
{\left[{ }^{\circ},{ }^{\prime} \prime \prime\right.}\end{array}$ & $z$ & $\begin{array}{c}\sigma \\
{\left[\mathrm{km} \mathrm{s}^{-1}\right]}\end{array}$ & $\begin{array}{c}M_{\text {vir }} \\
{\left[h^{-1} M_{\odot}\right]}\end{array}$ & $\begin{array}{c}R_{\mathrm{vir}} \\
{\left[h^{-1} \mathrm{Mpc}\right]}\end{array}$ & $\begin{array}{c}R_{200} \\
{\left[h^{-1} \mathrm{Mpc}\right]}\end{array}$ \\
\hline 155818.4 & +271612.0 & 0.090 & 1070.73 & $0.221 \times 10^{16}$ & 2.766 & 2.649 \\
\hline $\begin{array}{lll}15 & 10 & 1.7\end{array}$ & +332824.2 & 0.110 & 844.46 & $0.841 \times 10^{15}$ & 1.691 & 2.089 \\
\hline 132516.7 & +57371.2 & 0.116 & 747.53 & $0.772 \times 10^{15}$ & 1.980 & 1.850 \\
\hline 101955.6 & +405923.3 & 0.092 & 899.02 & $0.747 \times 10^{15}$ & 1.325 & 2.224 \\
\hline 164238.6 & +272624.0 & 0.104 & 983.57 & $0.145 \times 10^{16}$ & 2.151 & 2.434 \\
\hline 142830.8 & +564818.7 & 0.106 & 776.22 & $0.875 \times 10^{15}$ & 2.081 & 1.921 \\
\hline 102746.8 & +103226.9 & 0.109 & 673.64 & $0.438 \times 10^{15}$ & 1.383 & 1.667 \\
\hline 153950.6 & +304236.0 & 0.097 & 751.73 & $0.647 \times 10^{15}$ & 1.642 & 1.860 \\
\hline 143520.0 & +551058.1 & 0.140 & 910.40 & $0.121 \times 10^{16}$ & 2.095 & 2.253 \\
\hline 85415.1 & +29330.6 & 0.085 & 670.67 & $0.409 \times 10^{15}$ & 1.305 & 1.659 \\
\hline 145844.4 & +473224.0 & 0.085 & 603.92 & $0.450 \times 10^{15}$ & 1.767 & 1.494 \\
\hline 8189.8 & +543539.1 & 0.118 & 438.81 & $0.216 \times 10^{15}$ & 1.607 & 1.086 \\
\hline 123313.9 & +67712.0 & 0.105 & 1000.66 & $0.202 \times 10^{16}$ & 2.888 & 2.476 \\
\hline 9130.1 & +553916.6 & 0.116 & 979.65 & $0.547 \times 10^{15}$ & 0.817 & 2.424 \\
\hline 85451.5 & +0 5050.3 & 0.108 & 685.18 & $0.525 \times 10^{15}$ & 1.604 & 1.695 \\
\hline 152411.8 & +295122.0 & 0.114 & 1219.20 & $0.215 \times 10^{16}$ & 2.072 & 3.017 \\
\hline 152432.0 & +294338.6 & 0.112 & 1100.42 & $0.203 \times 10^{16}$ & 2.404 & 2.723 \\
\hline 154741.5 & +331953.0 & 0.114 & 518.23 & $0.285 \times 10^{15}$ & 1.519 & 1.282 \\
\hline 92326.9 & +8 392.5 & 0.129 & 844.89 & $0.996 \times 10^{15}$ & 2.001 & 2.091 \\
\hline 115351.2 & +152635.9 & 0.113 & 748.88 & $0.551 \times 10^{15}$ & 1.409 & 1.853 \\
\hline 162031.1 & +295327.6 & 0.097 & 853.98 & $0.103 \times 10^{16}$ & 2.030 & 2.113 \\
\hline 16319.8 & +252713.3 & 0.088 & 582.84 & $0.271 \times 10^{15}$ & 1.143 & 1.442 \\
\hline 145437.1 & +542523.2 & 0.100 & 630.37 & $0.423 \times 10^{15}$ & 1.525 & 1.560 \\
\hline 213027.0 & -0024.5 & 0.135 & 555.00 & $0.317 \times 10^{15}$ & 1.478 & 1.373 \\
\hline $\begin{array}{lll}15 & 1933.7\end{array}$ & +4 2016.8 & 0.103 & 664.20 & $0.361 \times 10^{15}$ & 1.173 & 1.643 \\
\hline 21494.6 & $\begin{array}{lll}-8 & 10 & 49.4\end{array}$ & 0.133 & 880.13 & $0.857 \times 10^{15}$ & 1.586 & 2.178 \\
\hline 8507.1 & +293252.1 & 0.104 & 730.39 & $0.707 \times 10^{15}$ & 1.899 & 1.807 \\
\hline 85457.5 & +352451.8 & 0.146 & 1271.75 & $0.245 \times 10^{16}$ & 2.170 & 3.147 \\
\hline 142921.1 & +23629.9 & 0.138 & 716.15 & $0.493 \times 10^{15}$ & 1.379 & 1.772 \\
\hline 114033.9 & +102141.0 & 0.105 & 412.45 & $0.143 \times 10^{15}$ & 1.201 & 1.021 \\
\hline 111348.5 & -02430.6 & 0.100 & 561.71 & $0.369 \times 10^{15}$ & 1.677 & 1.390 \\
\hline 164740.6 & +295518.1 & 0.099 & 1004.14 & $0.103 \times 10^{16}$ & 1.470 & 2.485 \\
\hline 9756.8 & +52482.9 & 0.099 & 732.91 & $0.462 \times 10^{15}$ & 1.232 & 1.813 \\
\hline 10934.3 & +444238.5 & 0.146 & 1080.56 & $0.161 \times 10^{16}$ & 1.974 & 2.674 \\
\hline 1080.4 & +3815.9 & 0.112 & 574.80 & $0.397 \times 10^{15}$ & 1.725 & 1.422 \\
\hline 103548.9 & +36558.6 & 0.123 & 462.89 & $0.169 \times 10^{15}$ & 1.134 & 1.145 \\
\hline 14746.5 & +14011.9 & 0.135 & 658.33 & $0.458 \times 10^{15}$ & 1.515 & 1.629 \\
\hline 112024.7 & +47926.3 & 0.112 & 605.14 & $0.425 \times 10^{15}$ & 1.664 & 1.497 \\
\hline 104134.6 & -03653.6 & 0.135 & 793.10 & $0.753 \times 10^{15}$ & 1.717 & 1.962 \\
\hline 22519.2 & -8445.3 & 0.054 & 479.32 & $0.131 \times 10^{15}$ & 0.817 & 1.186 \\
\hline 02822.5 & +135223.5 & 0.141 & 578.78 & $0.496 \times 10^{15}$ & 2.125 & 1.432 \\
\hline 121252.5 & +6348.2 & 0.137 & 580.40 & $0.353 \times 10^{15}$ & 1.502 & 1.436 \\
\hline 124720.0 & +0 839.1 & 0.089 & 779.99 & $0.680 \times 10^{15}$ & 1.604 & 1.930 \\
\hline 144733.3 & +33238.4 & 0.087 & 314.85 & $0.901 \times 10^{14}$ & 1.303 & 0.779 \\
\hline 15291.3 & +304620.6 & 0.105 & 647.03 & $0.413 \times 10^{15}$ & 1.414 & 1.601 \\
\hline 82929.4 & +39927.7 & 0.092 & 659.36 & $0.417 \times 10^{15}$ & 1.376 & 1.632 \\
\hline 113.3 & -95734.6 & 0.150 & 776.20 & $0.765 \times 10^{15}$ & 1.820 & 1.921 \\
\hline 132154.0 & +573221.5 & 0.118 & 379.97 & $0.121 \times 10^{15}$ & 1.201 & 0.940 \\
\hline 205142.2 & -048.0 & 0.108 & 468.59 & $0.180 \times 10^{15}$ & 1.177 & 1.159 \\
\hline 212636.9 & -63913.0 & 0.124 & 513.26 & $0.291 \times 10^{15}$ & 1.584 & 1.270 \\
\hline 1248.3 & +4 1933.2 & 0.136 & 737.57 & $0.654 \times 10^{15}$ & 1.724 & 1.825 \\
\hline 151646.1 & -0542.9 & 0.118 & 877.76 & $0.118 \times 10^{16}$ & 2.204 & 2.172 \\
\hline 10581.5 & +41247.8 & 0.129 & 919.45 & $0.867 \times 10^{15}$ & 1.471 & 2.275 \\
\hline 112126.4 & +534456.8 & 0.104 & 694.49 & $0.536 \times 10^{15}$ & 1.592 & 1.718 \\
\hline 81452.7 & +65742.8 & 0.110 & 575.06 & $0.283 \times 10^{15}$ & 1.225 & 1.423 \\
\hline 124840.0 & +62373.0 & 0.104 & 640.81 & $0.294 \times 10^{15}$ & 1.025 & 1.586 \\
\hline 164537.9 & +271845.0 & 0.101 & 511.66 & $0.188 \times 10^{15}$ & 1.029 & 1.266 \\
\hline 91557.8 & +5290.6 & 0.142 & 624.91 & $0.445 \times 10^{15}$ & 1.633 & 1.546 \\
\hline
\end{tabular}


V. Coenda and H. Muriel: Galaxy properties in clusters, Online Material p 3

Table A.2. continued.

\begin{tabular}{|c|c|c|c|c|c|c|}
\hline $\begin{array}{l}\alpha(\mathrm{J} 2000.00) \\
{[\mathrm{h} \mathrm{m} \mathrm{s}]}\end{array}$ & $\begin{array}{l}\delta(\mathrm{J} 2000.00) \\
{\left[{ }^{\circ}{ }^{\prime}{ }^{\prime \prime}\right]}\end{array}$ & $z$ & $\begin{array}{c}\sigma \\
{\left[\mathrm{km} \mathrm{s}^{-1}\right]}\end{array}$ & $\begin{array}{c}M_{\mathrm{vir}} \\
{\left[h^{-1} M_{\odot}\right]}\end{array}$ & $\begin{array}{c}R_{\mathrm{vir}} \\
{\left[h^{-1} \mathrm{Mpc}\right]}\end{array}$ & $\begin{array}{c}R_{200} \\
{\left[h^{-1} \mathrm{Mpc}\right]}\end{array}$ \\
\hline 102015.3 & $\begin{array}{llll}+39 & 18 & 13.3\end{array}$ & 0.147 & 445.84 & $0.169 \times 10^{15}$ & 1.218 & 1.103 \\
\hline 21478.5 & -75330.8 & 0.130 & 761.77 & $0.447 \times 10^{15}$ & 1.104 & 1.885 \\
\hline 214116.2 & -8239.2 & 0.129 & 826.61 & $0.702 \times 10^{15}$ & 1.474 & 2.045 \\
\hline 15108.9 & +25948.8 & 0.093 & 571.04 & $0.320 \times 10^{15}$ & 1.408 & 1.413 \\
\hline 114616.8 & +11111.7 & 0.112 & 397.07 & $0.147 \times 10^{15}$ & 1.337 & 0.983 \\
\hline 22582.6 & +14948.6 & 0.094 & 433.07 & $0.103 \times 10^{15}$ & 0.785 & 1.072 \\
\hline $1543 \quad 12.4$ & +5526.2 & 0.106 & 835.13 & $0.932 \times 10^{15}$ & 1.916 & 2.066 \\
\hline 114217.5 & +101730.8 & 0.117 & 435.71 & $0.134 \times 10^{15}$ & 1.013 & 1.078 \\
\hline 9637.6 & +10195.2 & 0.134 & 762.70 & $0.782 \times 10^{15}$ & 1.928 & 1.887 \\
\hline 114453.8 & +51161.2 & 0.129 & 804.40 & $0.881 \times 10^{15}$ & 1.953 & 1.990 \\
\hline 125558.8 & +62848.5 & 0.105 & 653.23 & $0.303 \times 10^{15}$ & 1.018 & 1.616 \\
\hline 0217.6 & -0 5531.4 & 0.111 & 623.97 & $0.375 \times 10^{15}$ & 1.382 & 1.544 \\
\hline $12 \quad 151.9$ & -24125.8 & 0.110 & 347.44 & $0.823 \times 10^{14}$ & 0.978 & 0.860 \\
\hline 102925.1 & +373748.0 & 0.108 & 304.95 & $0.108 \times 10^{15}$ & 1.666 & 0.755 \\
\hline 164744.9 & +291055.2 & 0.134 & 1077.76 & $0.179 \times 10^{16}$ & 2.205 & 2.667 \\
\hline $1523 \quad 36.5$ & +31117.4 & 0.074 & 909.39 & $0.120 \times 10^{16}$ & 2.082 & 2.250 \\
\hline 22956.2 & -75050.6 & 0.116 & 555.93 & $0.355 \times 10^{15}$ & 1.647 & 1.376 \\
\hline 115358.9 & +9 3929.9 & 0.103 & 766.07 & $0.557 \times 10^{15}$ & 1.360 & 1.896 \\
\hline 75939.4 & +415024.0 & 0.132 & 647.49 & $0.477 \times 10^{15}$ & 1.631 & 1.602 \\
\hline 153956.0 & -21147.8 & 0.150 & 941.81 & $0.129 \times 10^{16}$ & 2.088 & 2.330 \\
\hline 114345.5 & +462056.4 & 0.114 & 496.34 & $0.290 \times 10^{15}$ & 1.689 & 1.228 \\
\hline 142914.4 & +335923.6 & 0.130 & 919.26 & $0.147 \times 10^{16}$ & 2.500 & 2.275 \\
\hline 114859.9 & +513654.4 & 0.131 & 883.70 & $0.114 \times 10^{16}$ & 2.095 & 2.187 \\
\hline 151639.5 & +24712.8 & 0.111 & 466.11 & $0.173 \times 10^{15}$ & 1.140 & 1.153 \\
\hline $1414 \quad 12.3$ & +6523.0 & 0.109 & 639.74 & $0.469 \times 10^{15}$ & 1.642 & 1.583 \\
\hline 21401.0 & -8553.5 & 0.132 & 709.70 & $0.653 \times 10^{15}$ & 1.860 & 1.756 \\
\hline 104937.8 & +3 3851.4 & 0.151 & 759.96 & $0.816 \times 10^{15}$ & 2.025 & 1.880 \\
\hline 234928.7 & +15146.7 & 0.114 & 645.35 & $0.361 \times 10^{15}$ & 1.243 & 1.597 \\
\hline 11441.4 & -14527.7 & 0.106 & 647.10 & $0.400 \times 10^{15}$ & 1.370 & 1.601 \\
\hline 134057.8 & +3953.3 & 0.115 & 483.52 & $0.270 \times 10^{15}$ & 1.653 & 1.196 \\
\hline 16733.4 & +231318.1 & 0.088 & 420.68 & $0.132 \times 10^{15}$ & 1.069 & 1.041 \\
\hline 154234.4 & +414913.1 & 0.141 & 701.91 & $0.616 \times 10^{15}$ & 1.793 & 1.737 \\
\hline 143033.6 & +24403.4 & 0.134 & 873.70 & $0.123 \times 10^{16}$ & 2.306 & 2.162 \\
\hline 103017.4 & +41828.7 & 0.091 & 483.52 & $0.180 \times 10^{15}$ & 1.105 & 1.196 \\
\hline 85410.1 & +233433.2 & 0.112 & 481.69 & $0.222 \times 10^{15}$ & 1.372 & 1.192 \\
\hline 75325.2 & +341632.5 & 0.139 & 410.75 & $0.151 \times 10^{15}$ & 1.286 & 1.016 \\
\hline 83916.7 & +82250.9 & 0.132 & 815.42 & $0.996 \times 10^{15}$ & 2.148 & 2.018 \\
\hline 16344.8 & +405559.9 & 0.136 & 591.23 & $0.323 \times 10^{15}$ & 1.326 & 1.463 \\
\hline 103135.7 & +35315.8 & 0.122 & 928.83 & $0.130 \times 10^{16}$ & 2.161 & 2.298 \\
\hline $951 \quad 14.2$ & +8 1651.6 & 0.142 & 737.42 & $0.729 \times 10^{15}$ & 1.921 & 1.825 \\
\hline 102437.1 & +501328.9 & 0.156 & 487.80 & $0.378 \times 10^{15}$ & 2.275 & 1.207 \\
\hline 103652.7 & +445235.0 & 0.124 & 763.93 & $0.593 \times 10^{15}$ & 1.457 & 1.890 \\
\hline 135644.8 & +445411.9 & 0.126 & 717.58 & $0.711 \times 10^{15}$ & 1.981 & 1.776 \\
\hline 101622.8 & +333817.5 & 0.129 & 847.43 & $0.110 \times 10^{16}$ & 2.203 & 2.097 \\
\hline 1486.7 & +63334.9 & 0.112 & 601.25 & $0.385 \times 10^{15}$ & 1.528 & 1.488 \\
\hline 112730.3 & +0919.4 & 0.131 & 517.61 & $0.194 \times 10^{15}$ & 1.039 & 1.281 \\
\hline 84447.3 & +274120.8 & 0.085 & 483.87 & $0.160 \times 10^{15}$ & 0.983 & 1.197 \\
\hline $11 \quad 126.7$ & +304041.9 & 0.074 & 623.97 & $0.326 \times 10^{15}$ & 1.202 & 1.544 \\
\hline 215036.1 & -75328.0 & 0.122 & 639.67 & $0.351 \times 10^{15}$ & 1.229 & 1.583 \\
\hline $13 \quad 1424.7$ & +621945.8 & 0.135 & 580.22 & $0.341 \times 10^{15}$ & 1.452 & 1.436 \\
\hline 11434.0 & +11136.5 & 0.153 & 679.73 & $0.363 \times 10^{15}$ & 1.128 & 1.682 \\
\hline 122732.3 & +492844.0 & 0.119 & 560.16 & $0.261 \times 10^{15}$ & 1.194 & 1.386 \\
\hline 10329.4 & +531912.7 & 0.135 & 557.49 & $0.330 \times 10^{15}$ & 1.520 & 1.379 \\
\hline 92430.8 & +75656.0 & 0.105 & 423.20 & $0.137 \times 10^{15}$ & 1.096 & 1.047 \\
\hline 11424.8 & +483642.8 & 0.111 & 581.89 & $0.337 \times 10^{15}$ & 1.425 & 1.440 \\
\hline 143750.0 & +483630.6 & 0.122 & 782.47 & $0.769 \times 10^{15}$ & 1.801 & 1.936 \\
\hline 84333.9 & +385556.3 & 0.121 & 484.42 & $0.218 \times 10^{15}$ & 1.330 & 1.199 \\
\hline 132118.9 & -04343.7 & 0.108 & 627.17 & $0.271 \times 10^{15}$ & 0.987 & 1.552 \\
\hline 12139.0 & +58138.6 & 0.104 & 806.22 & $0.897 \times 10^{15}$ & 1.980 & 1.995 \\
\hline
\end{tabular}


V. Coenda and H. Muriel: Galaxy properties in clusters, Online Material p 4

Table A.2. continued.

\begin{tabular}{|c|c|c|c|c|c|c|}
\hline $\begin{array}{l}\alpha(\mathrm{J} 2000.00) \\
{[\mathrm{h} \mathrm{m} \mathrm{s}]}\end{array}$ & $\begin{array}{l}\delta(\mathrm{J} 2000.00) \\
{\left[{ }^{\circ},{ }^{\prime} \prime \prime\right.}\end{array}$ & $z$ & $\begin{array}{c}\sigma \\
{\left[\mathrm{km} \mathrm{s}^{-1}\right]}\end{array}$ & $\begin{array}{c}M_{\text {vir }} \\
{\left[h^{-1} M_{\odot}\right]}\end{array}$ & $\begin{array}{c}R_{\mathrm{vir}} \\
{\left[h^{-1} \mathrm{Mpc}\right]}\end{array}$ & $\begin{array}{c}R_{200} \\
{\left[h^{-1} \mathrm{Mpc}\right]}\end{array}$ \\
\hline 104558.5 & +12656.0 & 0.105 & 375.80 & $0.143 \times 10^{15}$ & 1.451 & 0.930 \\
\hline 10451.5 & +5856.0 & 0.116 & 512.04 & $0.226 \times 10^{15}$ & 1.239 & 1.267 \\
\hline 162456.8 & +283341.4 & 0.145 & 887.74 & $0.724 \times 10^{15}$ & 1.317 & 2.197 \\
\hline 9468.6 & +34640.8 & 0.119 & 545.97 & $0.317 \times 10^{15}$ & 1.523 & 1.351 \\
\hline 164746.2 & +29109.5 & 0.134 & 655.71 & $0.600 \times 10^{15}$ & 2.001 & 1.622 \\
\hline 113718.2 & +5782.8 & 0.117 & 560.11 & $0.201 \times 10^{15}$ & 0.9 acommentary 20 & 1.386 \\
\hline 134813.1 & +574138.0 & 0.127 & 1090.14 & $0.193 \times 10^{16}$ & 2.323 & 2.697 \\
\hline 04549.8 & -0511.4 & 0.105 & 567.44 & $0.311 \times 10^{15}$ & 1.386 & 1.404 \\
\hline 1214.5 & +151235.6 & 0.109 & 529.12 & $0.179 \times 10^{15}$ & 0.915 & 1.309 \\
\hline 142724.5 & +55451.1 & 0.131 & 713.57 & $0.624 \times 10^{15}$ & 1.757 & 1.766 \\
\hline 10751.3 & +62 302.2 & 0.137 & 560.11 & $0.187 \times 10^{15}$ & 0.853 & 1.386 \\
\hline 164140.6 & +22054.4 & 0.151 & 850.87 & $0.913 \times 10^{15}$ & 1.807 & 2.105 \\
\hline 132336.1 & +44240.3 & 0.134 & 794.57 & $0.724 \times 10^{15}$ & 1.643 & 1.966 \\
\hline 8560.8 & +482910.3 & 0.124 & 668.77 & $0.421 \times 10^{15}$ & 1.351 & 1.655 \\
\hline 152115.7 & +32512.1 & 0.111 & 827.47 & $0.826 \times 10^{15}$ & 1.729 & 2.047 \\
\hline 135920.8 & +492651.4 & 0.106 & 553.87 & $0.289 \times 10^{15}$ & 1.353 & 1.370 \\
\hline 92659.7 & +542236.1 & 0.125 & 673.39 & $0.276 \times 10^{15}$ & 0.872 & 1.666 \\
\hline 153629.8 & -15748.2 & 0.145 & 716.22 & $0.619 \times 10^{15}$ & 1.730 & 1.772 \\
\hline 112135.4 & +352324.7 & 0.103 & 591.23 & $0.395 \times 10^{15}$ & 1.619 & 1.463 \\
\hline 114820.4 & +10218.3 & 0.113 & 999.27 & $0.158 \times 10^{16}$ & 2.269 & 2.473 \\
\hline 10488.8 & +312831.1 & 0.115 & 623.88 & $0.302 \times 10^{15}$ & 1.112 & 1.544 \\
\hline 142349.9 & +6 1432.3 & 0.113 & 463.77 & $0.224 \times 10^{15}$ & 1.495 & 1.148 \\
\hline 144818.4 & +33144.4 & 0.124 & 741.07 & $0.522 \times 10^{15}$ & 1.362 & 1.834 \\
\hline 817.0 & +175845.1 & 0.144 & 454.09 & $0.109 \times 10^{15}$ & 0.756 & 1.124 \\
\hline 111333.7 & +372227.8 & 0.102 & 556.72 & $0.300 \times 10^{15}$ & 1.386 & 1.378 \\
\hline 94030.1 & +22835.4 & 0.151 & 865.54 & $0.813 \times 10^{15}$ & 1.556 & 2.142 \\
\hline 9118.4 & +58168.0 & 0.097 & 839.35 & $0.547 \times 10^{15}$ & 1.113 & 2.077 \\
\hline 144258.5 & +551055.2 & 0.105 & 435.71 & $0.111 \times 10^{15}$ & 0.840 & 1.078 \\
\hline 84436.5 & +292112.6 & 0.099 & 505.37 & $0.171 \times 10^{15}$ & 0.960 & 1.250 \\
\hline 10381.8 & +414625.7 & 0.124 & 567.73 & $0.289 \times 10^{15}$ & 1.285 & 1.405 \\
\hline 145233.6 & +505522.1 & 0.131 & 560.11 & $0.313 \times 10^{15}$ & 1.430 & 1.386 \\
\hline 14625.0 & +6 353.1 & 0.113 & 477.41 & $0.239 \times 10^{15}$ & 1.501 & 1.181 \\
\hline 121649.1 & -3755.2 & 0.111 & 435.64 & $0.134 \times 10^{15}$ & 1.012 & 1.078 \\
\hline 23618.9 & +14940.7 & 0.112 & 475.07 & $0.236 \times 10^{15}$ & 1.500 & 1.175 \\
\hline 14225.6 & -101611.6 & 0.112 & 507.75 & $0.250 \times 10^{15}$ & 1.391 & 1.256 \\
\hline 141754.2 & +432317.2 & 0.105 & 430.73 & $0.168 \times 10^{15}$ & 1.296 & 1.066 \\
\hline 132348.6 & +1648.2 & 0.108 & 668.18 & $0.369 \times 10^{15}$ & 1.187 & 1.653 \\
\hline 84421.1 & +512421.2 & 0.097 & 496.13 & $0.186 \times 10^{15}$ & 1.081 & 1.228 \\
\hline $1151 \quad 10.7$ & -3141.2 & 0.091 & 212.72 & $0.268 \times 10^{14}$ & 0.850 & 0.526 \\
\hline 101935.7 & +14226.5 & 0.146 & 631.06 & $0.544 \times 10^{15}$ & 1.959 & 1.561 \\
\hline 121358.5 & +631316.0 & 0.133 & 541.44 & $0.170 \times 10^{15}$ & 0.831 & 1.340 \\
\hline 16208.2 & +42302.9 & 0.135 & 658.63 & $0.524 \times 10^{15}$ & 1.732 & 1.630 \\
\hline $1017 \quad 17.7$ & +84057.7 & 0.104 & 522.25 & $0.215 \times 10^{15}$ & 1.131 & 1.292 \\
\hline 113736.7 & +322640.6 & 0.103 & 510.52 & $0.174 \times 10^{15}$ & 0.959 & 1.263 \\
\hline 8280.9 & +281551.8 & 0.093 & 418.11 & $0.136 \times 10^{15}$ & 1.119 & 1.035 \\
\hline 91626.7 & +55515.2 & 0.134 & 754.05 & $0.637 \times 10^{15}$ & 1.606 & 1.866 \\
\hline 10935.2 & +7950.8 & 0.099 & 425.58 & $0.131 \times 10^{15}$ & 1.038 & 1.053 \\
\hline 11409.3 & +322320.4 & 0.131 & 818.31 & $0.852 \times 10^{15}$ & 1.825 & 2.025 \\
\hline 234755.1 & +145452.2 & 0.105 & 424.17 & $0.205 \times 10^{15}$ & 1.630 & 1.050 \\
\hline 102828.7 & +93858.6 & 0.106 & 581.90 & $0.382 \times 10^{15}$ & 1.619 & 1.440 \\
\hline 0521.1 & +15584.8 & 0.117 & 541.07 & $0.430 \times 10^{15}$ & 2.106 & 1.339 \\
\hline 9649.9 & +44645.8 & 0.126 & 727.16 & $0.475 \times 10^{15}$ & 1.288 & 1.799 \\
\hline 101158.3 & +333415.2 & 0.127 & 631.29 & $0.349 \times 10^{15}$ & 1.255 & 1.562 \\
\hline 9399.5 & +37401.6 & 0.148 & 835.21 & $0.588 \times 10^{15}$ & 1.208 & 2.067 \\
\hline 104352.0 & +1342.1 & 0.117 & 419.59 & $0.140 \times 10^{15}$ & 1.137 & 1.038 \\
\hline 131122.6 & +483045.4 & 0.140 & 807.27 & $0.970 \times 10^{15}$ & 2.134 & 1.997 \\
\hline 3559.3 & -0959.4 & 0.110 & 623.76 & $0.256 \times 10^{15}$ & 0.944 & 1.543 \\
\hline 121052.2 & +155535.0 & 0.107 & 553.89 & $0.262 \times 10^{15}$ & 1.227 & 1.371 \\
\hline 12199.3 & +633142.2 & 0.107 & 611.17 & $0.405 \times 10^{15}$ & 1.554 & 1.512 \\
\hline
\end{tabular}


V. Coenda and H. Muriel: Galaxy properties in clusters, Online Material p 5

Table A.2. continued.

\begin{tabular}{|c|c|c|c|c|c|c|}
\hline $\begin{array}{l}\alpha(\mathrm{J} 2000.00) \\
{[\mathrm{h} \mathrm{m} \mathrm{s}]}\end{array}$ & $\begin{array}{l}\delta(\mathrm{J} 2000.00) \\
{\left[{ }^{\circ}{ }^{\prime}{ }^{\prime}\right]}\end{array}$ & $z$ & $\begin{array}{c}\sigma \\
{\left[\mathrm{km} \mathrm{s}^{-1}\right]}\end{array}$ & $\begin{array}{c}M_{\mathrm{vir}} \\
{\left[h^{-1} M_{\odot}\right]}\end{array}$ & $\begin{array}{c}R_{\text {vir }} \\
{\left[h^{-1} \mathrm{Mpc}\right]}\end{array}$ & $\begin{array}{c}R_{200} \\
{\left[h^{-1} \mathrm{Mpc}\right]}\end{array}$ \\
\hline 102726.5 & +375237.6 & 0.107 & 403.63 & $0.163 \times 10^{15}$ & 1.435 & 0.999 \\
\hline 95053.3 & +284815.5 & 0.113 & 810.78 & $0.837 \times 10^{15}$ & 1.827 & 2.006 \\
\hline 12119.8 & +61049.8 & 0.138 & 559.20 & $0.240 \times 10^{15}$ & 1.101 & 1.384 \\
\hline 10250.7 & +495133.5 & 0.135 & 1120.42 & $0.153 \times 10^{16}$ & 1.743 & 2.772 \\
\hline 143339.1 & +61223.4 & 0.113 & 618.08 & $0.446 \times 10^{15}$ & 1.675 & 1.529 \\
\hline 114115.1 & +104315.6 & 0.105 & 636.26 & $0.436 \times 10^{15}$ & 1.545 & 1.574 \\
\hline 95352.6 & +121555.4 & 0.129 & 521.13 & $0.286 \times 10^{15}$ & 1.508 & 1.289 \\
\hline 102845.1 & +375238.3 & 0.107 & 541.02 & $0.421 \times 10^{15}$ & 2.062 & 1.339 \\
\hline 101045.3 & +33433.7 & 0.135 & 664.31 & $0.685 \times 10^{15}$ & 2.224 & 1.644 \\
\hline 102116.9 & +12130.1 & 0.129 & 537.06 & $0.341 \times 10^{15}$ & 1.695 & 1.329 \\
\hline 94638.1 & +29422.2 & 0.112 & 253.32 & $0.473 \times 10^{14}$ & 1.057 & 0.627 \\
\hline 13259.1 & +511856.5 & 0.121 & 554.46 & $0.284 \times 10^{15}$ & 1.323 & 1.372 \\
\hline 11916.5 & +144231.0 & 0.129 & 525.18 & $0.339 \times 10^{15}$ & 1.760 & 1.299 \\
\hline 82548.4 & +56048.2 & 0.138 & 648.50 & $0.350 \times 10^{15}$ & 1.195 & 1.605 \\
\hline 134819.9 & +574534.6 & 0.127 & 541.44 & $0.323 \times 10^{15}$ & 1.582 & 1.340 \\
\hline 225628.3 & -03253.9 & 0.110 & 430.58 & $0.123 \times 10^{15}$ & 0.948 & 1.065 \\
\hline 75941.8 & +284655.6 & 0.139 & 503.43 & $0.177 \times 10^{15}$ & 0.999 & 1.246 \\
\hline 143222.8 & +47638.2 & 0.109 & 311.13 & $0.698 \times 10^{14}$ & 1.034 & 0.770 \\
\hline 111516.5 & +534242.5 & 0.105 & 903.25 & $0.106 \times 10^{16}$ & 1.867 & 2.235 \\
\hline 231458.7 & +14524.4 & 0.083 & 517.16 & $0.212 \times 10^{15}$ & 1.135 & 1.280 \\
\hline $1011 \quad 11.4$ & +84131.9 & 0.097 & 385.05 & $0.140 \times 10^{15}$ & 1.357 & 0.953 \\
\hline 15270.2 & +294133.0 & 0.113 & 689.54 & $0.512 \times 10^{15}$ & 1.543 & 1.706 \\
\hline 155137.4 & +453316.2 & 0.124 & 693.77 & $0.441 \times 10^{15}$ & 1.314 & 1.717 \\
\hline 111949.4 & +551537.8 & 0.106 & 537.54 & $0.251 \times 10^{15}$ & 1.245 & 1.330 \\
\hline 8633.0 & +292855.6 & 0.128 & 458.29 & $0.204 \times 10^{15}$ & 1.390 & 1.134 \\
\hline 82055.8 & +75212.7 & 0.110 & 558.19 & $0.287 \times 10^{15}$ & 1.321 & 1.381 \\
\hline 14149.8 & -0826.9 & 0.139 & 1091.89 & $0.190 \times 10^{16}$ & 2.280 & 2.702 \\
\hline 112153.8 & +04155.0 & 0.102 & 654.86 & $0.422 \times 10^{15}$ & 1.410 & 1.620 \\
\hline 112019.8 & +47454.5 & 0.112 & 572.08 & $0.359 \times 10^{15}$ & 1.570 & 1.416 \\
\hline 104858.7 & +545322.9 & 0.145 & 672.72 & $0.557 \times 10^{15}$ & 1.765 & 1.665 \\
\hline 14598.5 & +471943.0 & 0.089 & 677.50 & $0.660 \times 10^{15}$ & 2.062 & 1.676 \\
\hline 102749.7 & +34043.7 & 0.074 & 819.40 & $0.960 \times 10^{15}$ & 2.051 & 2.027 \\
\hline 212958.2 & -0 1947.6 & 0.135 & 323.62 & $0.126 \times 10^{15}$ & 1.721 & 0.801 \\
\hline
\end{tabular}

